\title{
RESEARCH
}

Open Access

\section{MicroRNA-503-3p affects osteogenic differentiation of human adipose-derived stem cells by regulation of Wnt 2 and Wnt7b under cyclic strain}

Yadong Luo ${ }^{1,2+}$, Xu Ding ${ }^{1,2+}$, Huan $\mathrm{Ji}^{1,2+}$, Meng Li ${ }^{1,2}$, Haiyang Song ${ }^{1,2}$, Sheng $\mathrm{Li}^{1,2}$, Chenxing Wang ${ }^{1,2}$, Heming $\mathrm{Wu}^{1,2}$ and Hongming $\mathrm{Du}^{1,2^{*}}$ (D)

\begin{abstract}
Background: MicroRNAs (miRNAs) play a role in regulating osteogenic differentiation (OD) of mesenchymal stem cells by inhibiting mRNAs translation under cyclic strain. miR-503-3p was downregulated in OD of human adiposederived stem cells (hASCs) in vivo under cyclic strain in our previous study, while it might target the Wnt/ $\beta$-catenin (W- $\beta$ ) pathway. In this study, we explored miR-503-3p's role in OD of hASCs under cyclic strain.

Methods: OD of hASCs was induced by cyclic strain. Bioinformatic and dual luciferase analyses were used to confirm the relationship between Wnt2/Wnt7b and miR-503-3p. Immunofluorescence was used to detect the effect of miR503-3p on Wnt2/Wnt7b and $\beta$-catenin in hASCs transfected with miR-503-3p mimic and inhibitor. Mimic, inhibitor, and small interfering RNA (siRNA) transfected in hASCs to against Wnt2 and Wnt7b. Quantitative real-time PCR (RT-PCR) and western blot were used to examine the OD and W- $\beta$ pathway at the mRNA and protein levels, respectively.

Immunofluorescence was performed to locate $\beta$-catenin. ALP activity and calcium were detected by colorimetric assay.

Results: Results of immunophenotypes by flow cytometry and multi-lineage potential confirmed that the cultured cells were hASCs. Results of luciferase reporter assay indicated that miR-503-3p could regulate the expression levels of Wnt2 and Wnt7b by targeting their respective 3'-untranslated region (UTR). Under cyclic strain, gain- or loss-function of miR-503-3p studies by mimic and inhibitor revealed that decreasing expression of miR-503-3p could significantly bring about promotion of OD of hASCs, whereas increased expression of miR-503-3p inhibited OD. Furthermore, miR-503-3p high-expression reduced the activity of the W- $\beta$ pathway, as indicated by lowering expression of Wnt2 and Wnt7b, inactive $\beta$-catenin in miR-503-3p-treated hASCs. By contrast, miR-503-3p inhibition activated the W- $\beta$ pathway.

Conclusions: Collectively, our findings indicate that miR-503-3p is a negative factor in regulating W- $\beta$ pathway by Wnt2 and Wnt7b, which inhibit the OD of hASCs under cyclic strain.
\end{abstract}

Keywords: hASCs, Cyclic strain, Osteogenic differentiation, miR-503-3p, Wnt2, Wnt7b

\footnotetext{
* Correspondence: dhm_010@sina.com

†Yadong Luo, Xu Ding and Huan Ji contributed equally to this work.

'Department of Oral and Maxillofacial Surgery, Affiliated Stomatological Hospital of Nanjing Medical University, Hanzhong Road No.136, Nanjing

210029, Jiangsu Province, People's Republic of China

2Jiangsu Key Laboratory of Oral Disease, Nanjing Medical University, Nanjing

210029, Jiangsu Province, People's Republic of China
}

(c) The Author(s). 2020 Open Access This article is licensed under a Creative Commons Attribution 4.0 International License, which permits use, sharing, adaptation, distribution and reproduction in any medium or format, as long as you give appropriate credit to the original author(s) and the source, provide a link to the Creative Commons licence, and indicate if changes were made. The images or other third party material in this article are included in the article's Creative Commons licence, unless indicated otherwise in a credit line to the material. If material is not included in the article's Creative Commons licence and your intended use is not permitted by statutory regulation or exceeds the permitted use, you will need to obtain permission directly from the copyright holder. To view a copy of this licence, visit http://creativecommons.org/licenses/by/4.0/ The Creative Commons Public Domain Dedication waiver (http://creativecommons.org/publicdomain/zero/1.0/) applies to the data made available in this article, unless otherwise stated in a credit line to the data. 


\section{Introduction}

In 2001, hASCs were extracted for the first time by digestion of human adipose tissue [1]. hASCs have extensive proliferative potential and the ability to differentiate toward adipogenic, osteogenic, chondrogenic, and myogenic lineages [2, 3]. Compared with bone mesenchymal stem cells, that are normally utilized as seed cells in bone tissue engineering, the advantages of hASCs include a large number of cell sources, easy accession, and rapid proliferation [4]. More importantly, the osteogenic differentiation (OD) activity of ASCs does not decrease with the increase of donor age [5]. Current studies suggest that hASCs may be an important new source of seed cells in bone tissue engineering. An important aspect of bone regeneration that requires further study is to determine how in vitro OD of hASCs can be effectively promoted [6-8]. Furthermore, studies indicate that tensile strain can effectively promote OD of hASCs in vitro $[6,9]$; these results have been used to promote OD in bone regeneration [6-8].

The Wnt signaling pathway plays significant roles in regulating many vital biological processes, like embryonic formation and development, stem cells differentiation and maintenance $[10,11]$. The $\mathrm{W}-\beta$ signaling pathway plays an essential role in OD of stem cells. The activated W- $\beta$ pathway can bring about upregulation of specific genes for OD, such as runt-related transcription factor 2 (RUNX2), distal-less homeobox 5, thereby promoting OD [12]. At the same time, activation of the W$\beta$ pathway also plays a role in inhibition of adipogenic differentiation of stem cells $[13,14]$. Our previous experiments confirm that the $W-\beta$ signaling pathway could be activated in the process of OD of hASCs induced by cyclic tensile strain [6].

miRNAs, which are non-coding small RNAs, have the ability to bring about inhibition of the expression of target genes by suppressing the translation or degradation of target mRNAs. They play an essential role in human physiological and pathological processes, including apoptosis, cell division, differentiation, and organ development [15-17]. The previous report had indicated that the process of OD regulated by miRNAs through the W$\beta$ pathway [17]. Due to the species of miRNAs and the diversity of its targets, further research in this field is needed.

In prior studies carried out in our laboratory, miR503-3p expression in hASCs was downregulated during OD in vitro under tensile strain. Our data also showed that there was activation of the $\mathrm{W}-\beta$ pathway as well $[6$, 18]. Based on the result of bioinformation, Wnt2 and Wnt7b, which were activators of the $\mathrm{W}-\beta$ pathway, may be targets of miR-503-3p. Therefore, we hypothesized that W- $\beta$ pathway activated by Wnt2 and Wnt7b through downregulation of miR-503-3p was involved in
OD of hASCs induced by cyclic strain in vitro. In this study, we tested this hypothesis by a series of experiments, and the whole process was shown in Fig. 1. The result presented herein provided a theoretical and experimental basis in promoting OD of hASCs under cyclic strain for the application of mechanical factors in bone regeneration.

\section{Materials and methods}

The obtain method and characterization of hASCs

The obtain method and characterization of hASCs were illustrated in our previous study [18].

\section{Application of cyclic strain to hASCs}

The 4th passage of hASCs was plated at a density of $1.0 \times 10^{5}$ cells $/ \mathrm{ml}$ into BioFlex ${ }^{\mathrm{Tm}}$ plates (Flexcell, USA). Alpha-modified Eagle medium ( $\alpha$-MEM) (Gibco, USA) was used to culture the cells along with $10 \%$ fetal bovine serum (FBS) (Gibco, USA) in BioFlex ${ }^{\mathrm{Ta}}$ plates for $24 \mathrm{~h}$ under the condition of $37^{\circ} \mathrm{C}$ and $5 \% \mathrm{CO}_{2}$. After cells were adherent to silicone rubber in BioFlex ${ }^{\mathrm{Tw}}$ plates, they were loaded on uniaxial cyclic strain $(5 \%, 0.5 \mathrm{~Hz}, 2 \mathrm{~h} /$ days) for 6 days in $\alpha$-MEM with $10 \%$ FBS by Flexcell ${ }^{\circ}$ FX-5000 ${ }^{\text {ma }}$ Compression System (Flexcell, USA) under the condition of $37^{\circ} \mathrm{C}$ and $5 \% \mathrm{CO}_{2}$. The control group was maintained under identical culture conditions just without tension stimulation. Cells were used to detect by immunofluorescence, RT-PCR, and western blot analyses after 6-day cyclic strain loading.

\section{Bioinformatics analysis}

The sequence of miR-503-3p among species and their predicted binding sites to 3 '-UTR of Wnt2 and Wnt7b were analyzed using the following database: miRecords (http://mirecords.biolead.org/), TargetScan (http://www. targetscan.org), miRGator (http://genome.ewha.ac.kr/ miRGator/miRGator.html), miRWalk (http://mirwalk. umm.uni-heidelberg.de), and miRBase (http://www.mirbase.org).

\section{Transfecting of tools for nucleic acid expression}

The 4th passage hASCs were transfected with expression plasmid of Wnt2 (EX-Wnt2), siRNA for Wnt2 (siWnt2), expression plasmid of Wnt7b (EX-Wnt7b), siRNA for Wnt7b (siWnt7b), expression plasmid for negative control (EX-Ctrl), negative control of siRNA (siR-Ctrl), miR-503$3 p$ mimic, negative control of miR-503-3p mimic (miRCtrl mimic), miR-503-3p inhibitor, negative control of miR-503-3p inhibitor (miR-Ctrl inhibitor), Wnt2 3' UTRWild Type (wt-Wnt2), Wnt2 3' UTR-Mutant (mu-Wnt2), Wnt7b 3' UTR-Wild Type (wt-Wnt7b), and Wnt7b 3' UTR-Mutant (mu-Wnt7b) by Lipofectamine 2000 (Invitrogen, USA). All sequences for transfection in this section are listed in Table 1. The miR-503-3p mimic, inhibitor, 


\section{The whole process of experiments}

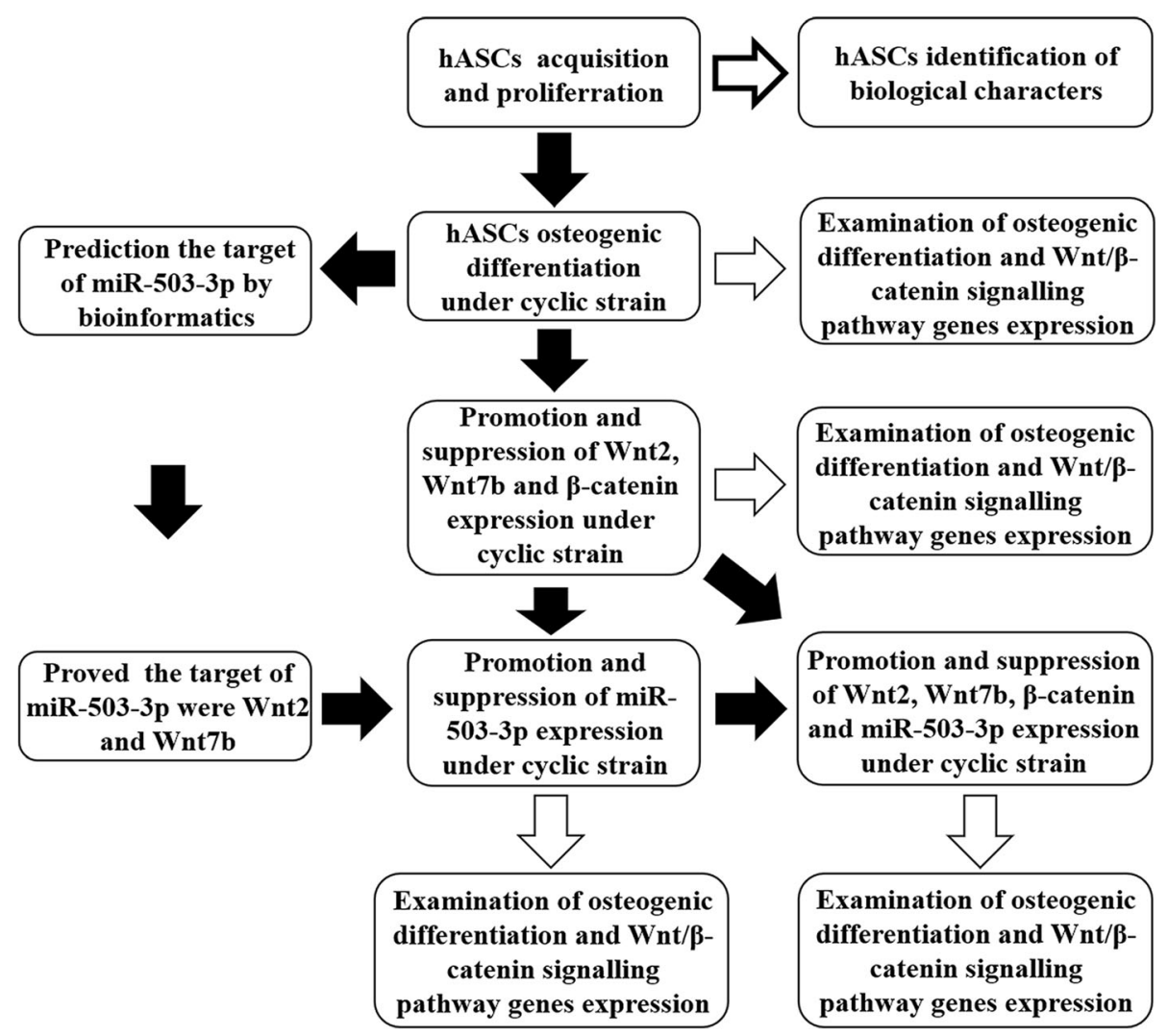

Fig. 1 Experimental flow diagram

siWnt2, siWnt7b, siR-Ctrl, and miR-Ctrl inhibitor and mimic, were obtained from GenePharma Corporation (Shanghai, China). The rest of mentioned tools for nucleic acid expression in this paragraph were obtained from GeneCopoeia Corporation (Guangzhou, China).

Lentiviral particles that contain shRNA control or shRNA targeting $\beta$-catenin (Mission Lentiviral Transduction Particles from Sigma-Aldrich, USA) were transduced into SKOV3 cells to control the $\beta$-catenin expression. After transducing, puromycin $(1.5 \mu \mathrm{M} / \mathrm{mL})$ was used to select the SKOV3 cells. Individual siRNA sequences (\#1: GCGUUUGGCUGAACCAUCA and \#2: UAAUGAGGACCUAUACUUA, Dharmacon) or a pool of 4 short interfering RNAs that targeted $\beta$-catenin (si $\beta$ catenin; Dharmacon, USA; siGenome SMART pool) were used to transfect performing by Dream FECT

Table 1 The constructed sequences used in this study

\begin{tabular}{|c|c|c|}
\hline Genes & Sequence & \\
\hline miR-503-3p mimic & & 5'-GGGGUAUUGUUUCCGCUGCCAGGdTdT-3' \\
\hline miR-Ctrl mimic & & 5'-UUGUACUACACAAAAGUACUGdTdT-3' \\
\hline miR-503-3p inhibitor & & 5'-CCUGGCAGCGGAAACAAUACCCCdTdT-3' \\
\hline miR-Ctrl inhibitor & & 5'-CAGUACUUUUGUGUAGUACAAdTdT-3' \\
\hline \multirow[t]{4}{*}{ siWnt2 siWnt7b } & Sense & 5'-GCCUUUGUUUAUGCCAUCUdTdT-3' \\
\hline & Antisense & 5'-AGAUGGCAUAAACAAAGGCdTdT-3' \\
\hline & Sense & 5'-CAGACCUGGUGUACAUUGAdTdT-3' \\
\hline & Antisense & 5'-UCAAUGUACACCAGGUCUGdTdT-3' \\
\hline \multirow[t]{2}{*}{ siR-Ctrl } & Sense & 5'-UUCUCCGAACGUGUCACGUdTdT-3' \\
\hline & Antisense & 5-ACGUGACACGUUCGGAGAAdTdT-3 \\
\hline
\end{tabular}


transfection reagent (Oz Biosciences, France). The control, scrambled siRNA pool (Dharmacon), was maintained.

\section{Dual luciferase reporter assay}

In this assay, insertion of the synthetic fragments of wtWnt2 or corresponding mu-Wnt2 that contained the predicted seed match site with miR-503-3p, were carried out between the Not I and Xho I cleavage sites of the psiCHECK-2 vector (Promega, USA), downstream of the Renilla luciferase reporter gene. HEK-293 T cells, on seeding into a white and opaque 96 -well plate at $70 \%$ confluence, were co-transfected with each reporter construct (pmirGLO-wt-Wnt2, pmirGLO-mu-Wnt2, pmirGLO-wtWnt7b, or pmirGLO-mu-Wnt7b) and Lv-miR-503-3p, Lv-miR-NC, Lv-ASO-503-3p, or LvASO-NC. According to the protocols of the manufacturer, Firefly and Renilla luciferase activity was detected $48 \mathrm{~h}$ after transfection with the Dual Luciferase Reporter Gene Assay Kit (Beyotime, China). This was done by normalization of firefly values Renilla luciferase.

\section{$\beta$-Catenin in hASCs detected by immunofluorescence}

After transfecting process mentioned in transfecting of tools for nucleic acid expression, the effect of miR-503-3p, Wnt2, and Wnt7b on the expression of $\beta$-catenin protein and their location were detected by immunofluorescence. After loading cyclic strain, samples were fixed by $4 \%$ paraformaldehyde in wells of BioFlex ${ }^{\text {Tw }}$ plates, then rinsing 3 times by PBS. At room temperature, $0.5 \%$ Triton X-100 (Sigma-Aldrich, USA) was added for transpiring them for 20 min. Goat serum (Beyotime, China) was used to block $\mathrm{hASCs}$ for $2 \mathrm{~h}$ and then incubated with primary antibodies specific for $\beta$-catenin (1:1000, Abcam, USA) overnight at $4{ }^{\circ} \mathrm{C}$. After it is rinsed thrice with PBS with Tween-20, cells were incubated with fluorescent $\mathrm{Cy} 3$ secondary antibodies (1:50, Proteintech, USA) for $1 \mathrm{~h}$ at $37^{\circ} \mathrm{C}$ in the dark. Sample photos were taken by fluorescence microscopy (ZEISS, Germany).

\section{Detection of gene expression with RT-PCR}

Total RNA isolated from cells by Trizol Reagent (Invitrogen, USA). PrimeScript RT Master Mix Perfect RealTime (TaKaRa, Japan) was used to synthesize cDNA from RNA. ABI 7300 Real-Time PCR System was used to carry out RT-PCR reactions (Applied Biosystems, UK). RUNX2, alkaline phosphatase (ALP), secreted protein acidic and cysteine rich (SPARC), Wnt2, Wnt7b, $\beta$ catenin, and glyceraldehyde-3-phosphate dehydrogenase (GAPDH) were detected by SYBR Premix ExTaq kit (TaKaRa, Japan). Primers designing and synthesis was done by the RiboBio Corporation (Guangzhou, China), and sequences of primers listed in Table 2. The results of RT-PCR analysis were calculated by $-\Delta \Delta$ Ct method.

\section{Western blot analysis for protein expression detection} Cell lysis buffer for Western and IP Kit (Beyotime, China) was used for total protein extraction; nuclear and cytoplasmic protein extraction kit was used to isolate the nuclear and cytoplasmic protein (Beyotime, China). Following centrifugation at $4{ }^{\circ} \mathrm{C}(12,000 \times g, 15 \mathrm{~min})$, bicinchoninic acid kit was used to quantify the protein concentrations (Beyotime, China). Protein samples were separated by sodium dodecyl sulfate polyacrylamide gel electrophoresis and transferred onto membranes (GE Healthcare Life Sciences, USA). Freshly prepared Trisbuffer saline (TBS) that contained 5\% non-fat milk was utilized for blocking the membranes for $2 \mathrm{~h}$ at room temperature. The blots were probed with primary antibodies at $4{ }^{\circ} \mathrm{C}$ overnight, in the dilution ratio $1: 1000$. The primary antibodies used in western blot were listed as follows: RUNX2 (Abcam, USA), ALP (Abcam, USA), SPARC (Abcam, USA), Wnt7b (R\&D Systems, USA), Wnt2 (R\&D Systems, USA), GAPDH (Cell Signaling Technology, USA), and $\beta$-catenin (Abcam, USA) and followed by washing of membranes thrice in TBS- $0.05 \%$ Tween 20, followed by incubation at room temperature with the corresponding secondary antibodies for $1 \mathrm{~h}$. Blots were then incubated in the dark with ECL (Thermo Fisher Scientific, Germany) and visualized by exposing to enhanced chemiluminescence reagents (GE Healthcare, USA). "Image J software 1.4.3.67" (National Institutes of Health, USA) was used to analyze the grayscales of blots.

\section{Detection of ALP activity and calcium with colorimetric assay}

The cells were lysed by cell lysis buffer for western and IP kit (Beyotime, China) following the manual of the manufacturers. Alkaline phosphatase assay kit (Beyotime, China) was used to detect the ALP activity in cell lysates. Samples and standards were added in 96-well plates, respectively. Then, para-nitrophenyl phosphate solution and ALP enzyme solution was added in sample and standard wells respectively and incubated for $10 \mathrm{~min}$ at $37^{\circ} \mathrm{C}$. Stop solution utilized to terminate the reaction. The 96-well plates analyzed with a microplate spectrophotometer (SpectraMax M2e, Molecular Devices, USA) at $405 \mathrm{~nm}$.

The calcium in cells was detected by calcium colorimetric assay kit (Beyotime, China). The cells were lysed by sample lysis solution in the kit. Following centrifugation at $4{ }^{\circ} \mathrm{C}(12,000 \times g, 5 \mathrm{~min})$, the supernatant was used for detecting. Fifty microliters standard and sample were added in 96-wells plates following the manual of manufactures. Test solution (test buffer to o-cresolphthalein complexone $=1: 1)$ was added to each well $(150 \mu \mathrm{l}$ per well) and incubated for $10 \mathrm{~min}$ at room temperature in the dark. The 96-well plates analyzed with a microplate 
Table 2 The sequence of primer used for RT-PCR in this study

\begin{tabular}{|c|c|c|c|}
\hline Gene & Accession No. & $5^{\prime}-3^{\prime}$ & $\operatorname{Tm}\left({ }^{\circ} \mathrm{C}\right)$ \\
\hline \multirow[t]{2}{*}{ RUNX2 } & NM_001015051 & F: TAGATAGTGATTGCGTTTGGCTATG & 60 \\
\hline & & R: CACTAAGAAATGTITCAAGGGTCC & 60 \\
\hline \multirow[t]{2}{*}{ ALP } & NM_003064 & F: GAAAGTCCTTCAAAGCTGGAGTCT & 60 \\
\hline & & R: TCTGGCACTCAGGTTTCTTGTATC & 60 \\
\hline \multirow[t]{2}{*}{ SPARC } & NM_001309443 & F: TGTGATCTAAATCCACTCCTTCCA & 60 \\
\hline & & R: ACAAACCATCCAAACATTTTAAACA & 60 \\
\hline \multirow[t]{2}{*}{ Wnt2 } & NM_004185 & F: GGGGCACGAGTGATCTGTG & 62 \\
\hline & & R: GCATGATGTCTGGGTAACGCT & 62 \\
\hline \multirow[t]{2}{*}{ Wnt7b } & NM_058238 & F: CACAGAAACTTTCGCAAGTGG & 60 \\
\hline & & R: GTACTGGCACTCGTTGATGC & 60 \\
\hline \multirow[t]{2}{*}{$\beta$-catenin } & XM_024453360 & F: TTGAACTGTTGAGGCGAAGAG & 60 \\
\hline & & R: ACTGAACACCGAGTTAGAGGAAT & 60 \\
\hline \multirow[t]{2}{*}{ GAPDH } & NM_001256799.2 & F: GAACGGGAAGCTCACTGG & 60 \\
\hline & & R: GCCTGCTTCACCACCTTCT & 60 \\
\hline
\end{tabular}

spectrophotometer (SpectraMax M2e, Molecular Devices, USA) at $575 \mathrm{~nm}$.

\section{Statistical analysis}

All data and statistical analysis were done with SPSS 22.0 (IBM-Corp., USA). Comparisons between groups were analyzed using the Student's $t$ tests (two-sided) or analysis of variance for experiments with more than two subgroups. The standard of statistical significance was $p<0.05$. All quantifiable results presented as the pattern of mean \pm standard error.

\section{Results}

Wnt2 and Wnt7b were targets of miR-503-3p

Based on the result of bioinformatics databases, the sequence of miR-503-3p in many species is conservative (Fig. 2a). Wnt2 3'-UTR and Wnt7b 3'-UTR could match with miR-503-3p (Fig. 2b, c).

To investigate the molecular mechanisms and determine whether miR-503-3p directly targeted Wnt2 and Wnt7b in hASCs, luciferase reporter gene assays were performed by constructed wt-Wnt2, mu-Wnt2, wtWnt7b, and mu-Wnt7b binding site mutagenesis of miR-503-3p. Then, above vectors and miR-503-3p mimic were co-transfected into HEK-293T cells.

The results showed that hASCs transfected with miR503-3p mimic and wt-Wnt2 significantly reduced the luciferase activity $1.63 \pm 0.39$-fold $(\mathrm{F})(p=0.024)$ when compared with hASCs transfected with miR-Ctrl mimic (MCM) and wt-Wnt2 (Fig. 2d). Moreover, the luciferase activity of hASCs transfected with miR-503-3p mimic and mut-Wnt2 had no statistical significance, compared with hASCs transfected with MCM and mu-Wnt2 ( $p=$ 0.377 ) (Fig. 2d), indicating the inhabitation of luciferase activity regulated by miR-503-3p mimic was broken by the mut-Wnt2.

The results showed that hASCs transfected with miR503-3p mimic containing wt-Wnt7b significantly reduced the luciferase activity $1.69 \pm 0.02-\mathrm{F}(p<0.001)$ in comparison with hASCs that were transfected with wtWnt7b and MCM (Fig. 2e). Moreover, the luciferase activity of hASCs transfected with miR-503-3p mimic containing mu-Wnt7b had no statistical significance, compared with hASCs transfected with mu-Wnt7b and MCM $(p=1.00)$ (Fig. 2e), indicating the inhabitation of luciferase activity regulated by miR-503-3p mimic was broken by the mu-Wnt7b.

Compared to hASCs transfected with MCM, Wnt2 and Wnt7b mRNA levels in hASCs transfected with miR-503$3 \mathrm{p}$ mimic were repressed $3.60 \pm 0.23-\mathrm{F}(p=0.003)$ and $2.32 \pm 0.04-\mathrm{F}(p=0.001)$ (Fig. $2 \mathrm{f}, \mathrm{g})$; the western blot analysis also suggested both Wnt2 and Wnt7b protein levels were also decreased $1.53 \pm 0.07-\mathrm{F}(p=0.005)$ and $1.80 \pm$ $0.07-\mathrm{F}(p=0.001)$ (Fig. 2h, i). This shows that Wnt2 and Wnt7b were target genes mediated by miR-503-3p at both transcriptional and translational levels.

\section{hASCs OD induced by cyclic strain}

Compared to hASCs without cyclic strain loading, the mRNA expression associated with OD (RUNX2, ALP, and SPARC; R, A, S) showed a significant increase with $2.22 \pm 0.01-\mathrm{F} \quad(p=0.002), 1.39 \pm 0.01-\mathrm{F} \quad(p=0.002)$, and $1.62 \pm 0.06-\mathrm{F}(p=0.002)$, respectively; the $\mathrm{W}-\beta$ pathway genes (Wnt2, Wnt7b, and $\beta$-catenin; W2, W7, $\beta$ ) showed an increase of $1.91 \pm 0.17-\mathrm{F} \quad(p=0.028), 1.72 \pm 0.05-\mathrm{F}$ $(p=0.034)$, and $1.47 \pm 0.11-\mathrm{F} \quad(p=0.014)$, respectively (Fig. 3a). 


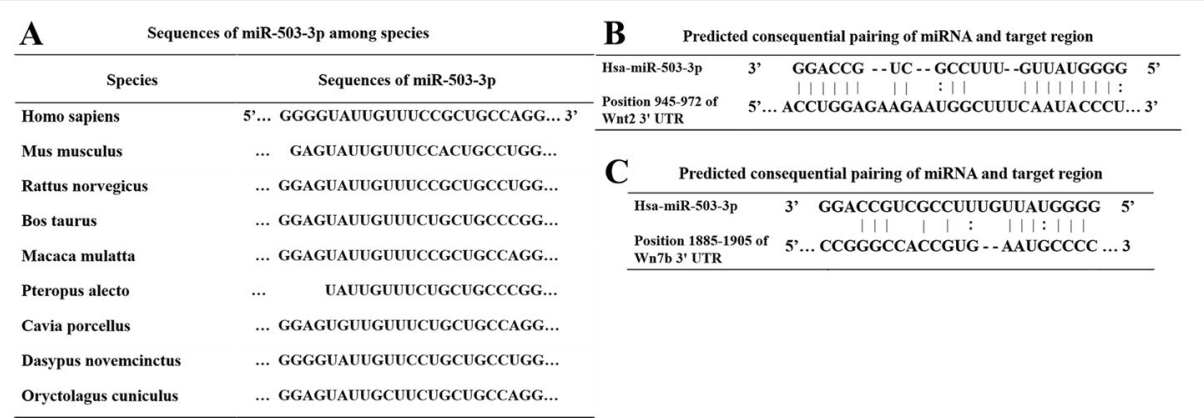
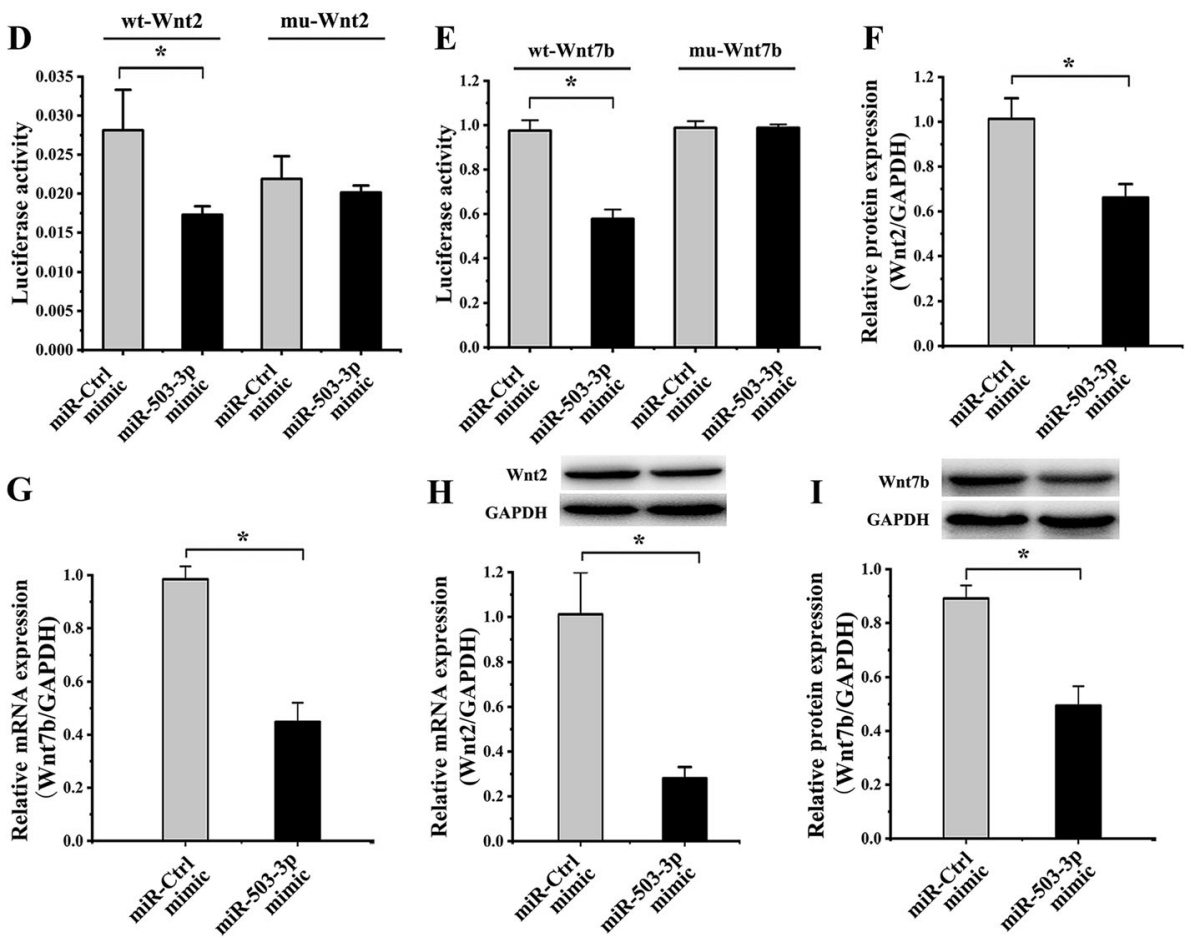

Fig. 2 Wnt2 and Wnt7b were targets of miR-503-3p. $\left({ }^{*} p<0.05\right.$, there were significant differences between these two groups) a The sequences of miR503-3p are highly conservative across species. b miR-503-3p might bind to Wnt2 3'-UTR. c miR-503-3p might bind to Wnt7b 3'-UTR. d The luciferase activity of hASCs co-transfected with miR-503-3p mimic and wt-Wnt2 significantly reduced, compared to the miR-Ctrl mimic and wt-Wnt2 group. The luciferase activity of hASCs co-transfected with miR-503-3p mimic and mut-Wnt2 had no significance, compared to miR-Ctrl mimic and mut-Wnt2 group. e The luciferase activity of hASCs co-transfected with miR-503-3p mimic and wt-Wnt7b significantly reduced, compared to the miR-Ctrl mimic and wt-Wnt7b group. The luciferase activity of hASCs co-transfected with miR-503-3p mimic and mut-Wnt7b had no significance, compared to miRCtrl mimic and mut-Wnt7b group. $\mathbf{f}$ Compared to the miR-Ctrl mimic group, Wnt2 mRNA levels decreased in hASCs transfected with miR-503-3p mimic as shown by real-time PCR. $\mathbf{g}$ Compared to the miR-Ctrl mimic group, Wnt7b mRNA levels decreased in hASCs transfected with miR-503-3p mimic as shown by real-time PCR. e Protein blots were listed in the upper column. Compared to the miR-Ctrl mimic group, Wnt2 protein levels decreased in hASCs transfected miR-503-3p mimic as shown by Western blot. f Protein blots were listed in the upper column. Compared to the miRCtrl mimic group, Wnt7b protein levels decreased in hASCs transfected miR-503-3p mimic as shown by western blot

In comparison to hASCs without cyclic strain loading, the $\mathrm{R}, \mathrm{A}, \mathrm{S}$ protein expression exhibited a significant increase $1.81 \pm 0.28-\mathrm{F}(p=0.002), 1.40 \pm 0.14-\mathrm{F}(p=0.030)$, and $1.74 \pm 0.22-\mathrm{F}(p=0.004)$, respectively; W2, W7, $\beta$ showed an increase of $1.50 \pm 0.14-\mathrm{F}(p=0.004), 1.36 \pm$ 0.11 -F $(p=0.006)$, and $1.63 \pm 0.08-\mathrm{F}(p<0.001)$, respectively (Fig. 3b).

Compared to hASCs without cyclic strain loading, the ALP activity showed a significant increase with $1.95 \pm$
$0.05-\mathrm{F}(p<0.001)$; the content of $\mathrm{Ca}^{2+}$ showed an increase of $2.13 \pm 0.17-\mathrm{F}(p=0.001)$ (Fig. 3c).

\section{Role of Wnt2 on hASCs osteogenic differentiation induced by cyclic strain}

For determining effects of transfection, Wnt2 expressions of hASCs, which were transfected with the EXWnt2, siWnt2, EX-Ctrl, and siR-Ctrl, were examined by immunofluorescence, real-time PCR, and western blot. 

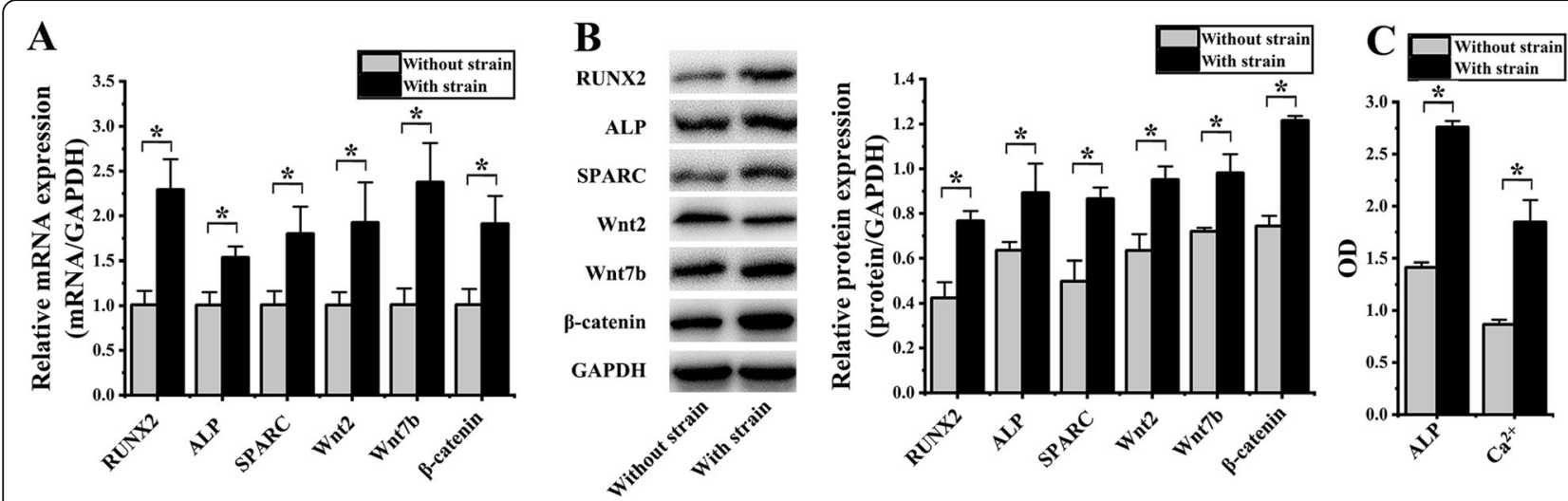

Fig. 3 The cyclic strain induced osteogenic differentiation of hASCs. $\left({ }^{*} p<0.05\right.$, there were significant differences between these two groups) a After cyclic strain loading for 6 days, the mRNA expression of RUNX2, ALP, SPARC, Wnt7b, and $\beta$-catenin were significantly increased, compared to hASCs without cyclic strain loading. b Protein blots were listed in the left column. After cyclic strain loading for 6 days, the protein expression of RUNX2, ALP, SPARC, Wnt7b, and $\beta$-catenin were significantly increased, compared to hASCs without cyclic strain loading. c After cyclic strain loading for 6 days, both the ALP activity and the content of $\mathrm{Ca}^{2+}$ were increased

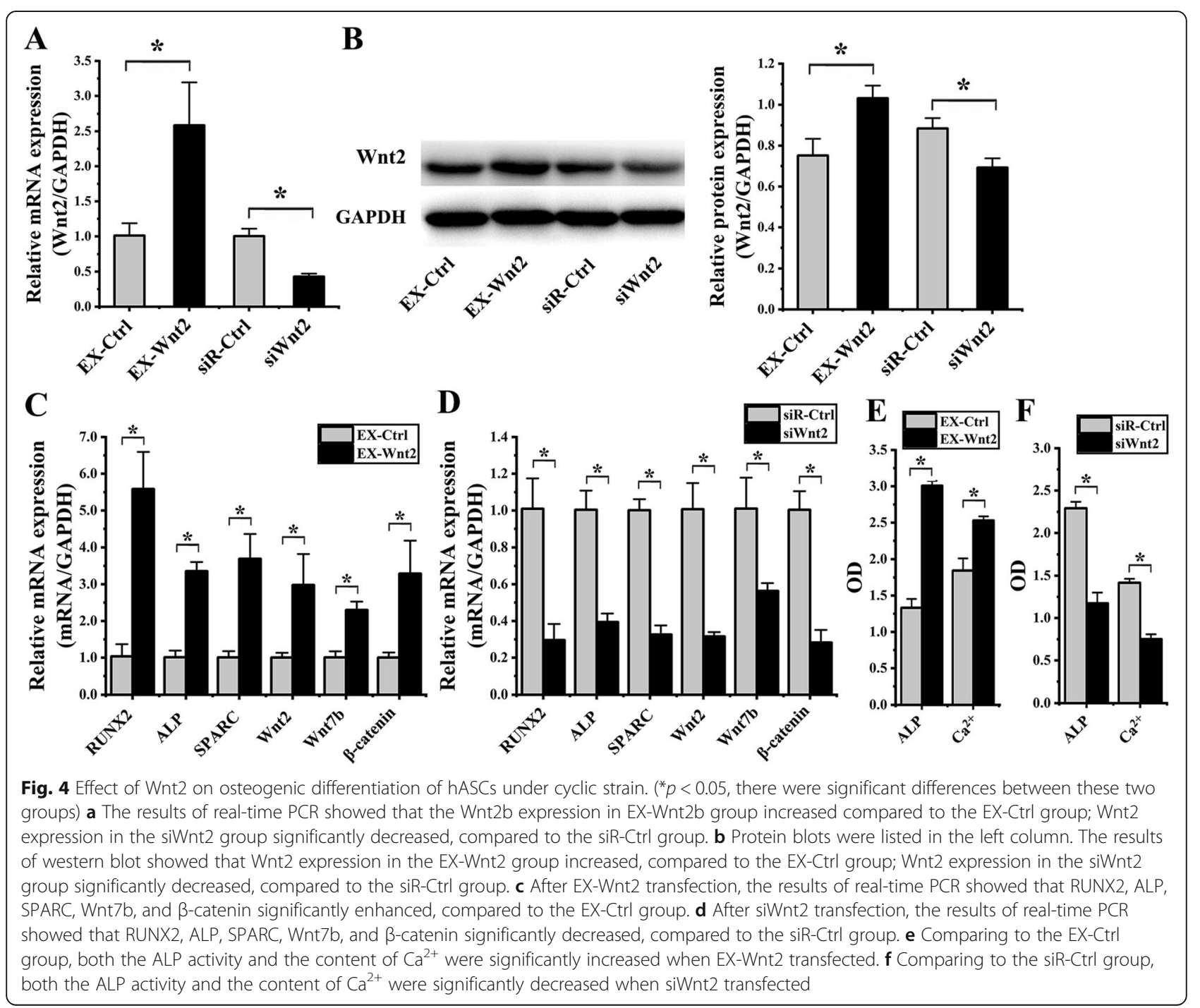


The results of RT-PCR analysis showed that the Wnt2 expression in EX-Wnt2 group increased $2.56 \pm 0.16$-F $(p=0.013)$ in comparison with EX-Ctrl group; Wnt2 expression in the siWnt2 group showed a significant decreased 2.34 \pm 0.45 -F $(p=0.001)$ in comparison with the siR-Ctrl group (Fig. 4a). The results of western blot analysis showed that Wnt2 expression in the EX-Wnt2 group showed an increase of $1.37 \pm 0.07-\mathrm{F}(p=0.009)$ in comparison with the EX-Ctrl group; Wnt2 expression in the siWnt2 group showed a significant decrease $1.28 \pm$ $0.03-\mathrm{F}(p=0.008)$ in comparison with the siR-Ctrl group (Fig. 4b).

Furthermore, RT-PCR analysis was used to determine effects of Wnt2 on the OD of hASCs after loading cyclic strain for 6 days. After EX-Wnt2 transfection, the results showed that $\mathrm{R}, \mathrm{A}, \mathrm{S}, \mathrm{W} 2, \mathrm{~W} 7, \beta$ significantly enhanced $5.39 \pm 2.14-\mathrm{F}(p=0.002), 3.32 \pm 0.80-\mathrm{F}(p<0.001), 3.66 \pm$ $1.20-\mathrm{F}(p=0.003), 2.96 \pm 0.44-\mathrm{F}(p=0.016), 2.28 \pm 0.62-\mathrm{F}$ $(p=0.001)$, and $3.27 \pm 0.49-\mathrm{F}(p=0.012)$, respectively, in comparison with the EX-Ctrl group (Fig. 4c). After siWnt2 transfected, the results showed that R, A, S, W2, W7, $\beta$ significantly reduced $3.41 \pm 0.09-\mathrm{F} \quad(p=0.003)$, $2.55 \pm 0.03-\mathrm{F}(p=0.001), 3.07 \pm 0.07-\mathrm{F}(p<0.001), 3.19 \pm$ $0.07-\mathrm{F}(p=0.001), 1.79 \pm 0.11-\mathrm{F}(p=0.011)$, and $3.55 \pm$ $0.10-\mathrm{F}(p=0.001)$ respectively, in comparison with the siR-Ctrl group (Fig. 4d). After EX-Wnt2 transfection, ALP activity and the content of $\mathrm{Ca}^{2+}$ significantly increased $2.26 \pm 0.15$-F $(p<0.001)$ and $1.37 \pm 0.17-\mathrm{F}(p=$ 0.003), respectively, in comparison with the EX-Ctrl group (Fig. 4e). After siWnt2 transfected, ALP activity and the content of $\mathrm{Ca}^{2+}$ significantly decreased $1.95 \pm$ $0.19-\mathrm{F}(p<0.001)$ and $1.87 \pm 0.11-\mathrm{F}(p<0.001)$, respectively, in comparison with the siR-Ctrl group (Fig. 4f).

All these results above suggested that overexpression of Wnt2 enhanced OD of hASCs, and knockdown of Wnt2 inhibited OD of hASCs.

\section{Role of Wnt7b on hASCs OD induced by cyclic strain} For determining effects of transfection, Wnt7b expressions of hASCs, which were transfected with the EXWnt7b, siR-Ctrl, EX-Ctrl, and siWnt7b were examined by real-time PCR, immunofluorescence, and western blot. The results of RT-PCR analysis indicated that the Wnt7b expression in EX-Wnt7b group increased $2.43 \pm$ $0.10-\mathrm{F}(p<0.001)$ in comparison with the EX-Ctrl group; Wnt7b expression in the siWnt7b group showed a significant decrease $2.98 \pm 0.02-\mathrm{F}(p<0.001)$ in comparison with the siR-Ctrl group (Fig. 5a). The results of western blot analysis showed that Wnt7b expression in the EXWnt7b group increased $1.54 \pm 0.12-\mathrm{F}(p=0.003)$ in comparison with the EX-Ctrl group; Wnt7b expression in the siWnt7b group showed a significant reduction $1.81 \pm 0.10-\mathrm{F}(p=0.003)$ in comparison with the siR-Ctrl group (Fig. 5b).
Furthermore, RT-PCR analysis was used to determine effects of Wnt7b on the OD of hASCs after loading cyclic strain for 6 days. After EX-Wnt7b transfection, the results showed that $\mathrm{R}, \mathrm{A}, \mathrm{S}, \mathrm{W} 2, \mathrm{~W} 7, \beta$ significantly enhanced $3.03 \pm 0.01-\mathrm{F}(p<0.001), 2.29 \pm 0.22-\mathrm{F}(p=0.007), 1.61 \pm$ $0.10-\mathrm{F}(p=0.027), 2.20 \pm 0.38-\mathrm{F}(p=0.034), 2.62 \pm 0.12-\mathrm{F}$ $(p<0.001)$, and $1.79 \pm 0.17-\mathrm{F}(p=0.013)$, respectively, compared to the EX-Ctrl group (Fig. 5c). After siWnt7b transfected, the results showed that $\mathrm{R}, \mathrm{A}, \mathrm{S}, \mathrm{W} 2, \mathrm{~W} 7, \beta$ significantly decreased $5.33 \pm 0.01-\mathrm{F}(p<0.001), 3.53 \pm$ $0.02-\mathrm{F}(p=0.001), 2.12 \pm 0.03-\mathrm{F}(p=0.018), 2.00 \pm 0.54-\mathrm{F}$ $(p=0.023), 4.24 \pm 0.40-\mathrm{F}(p<0.000)$, and $2.55 \pm 0.27-\mathrm{F}$ $(p<0.001)$, respectively, compared to the siR-Ctrl group (Fig. 5d). After EX-Wnt7b transfection, ALP activity and the content of $\mathrm{Ca}^{2+}$ significantly increased $1.38 \pm 0.08$-F $(p=0.008)$ and $1.59 \pm 0.04-\mathrm{F}(p<0.001)$, respectively, compared to the EX-Ctrl group (Fig. 5e). After was siWnt7b transfected, ALP activity and the content of $\mathrm{Ca} 2+$ significantly decreased $1.65 \pm 0.16-\mathrm{F}(p=0.001)$ and $1.80 \pm 0.15$ $\mathrm{F}(p=0.001)$, respectively, compared to the siR-Ctrl group (Fig. 5f).

All these results above suggested that overexpression of Wnt7b enhanced OD of hASCs, and knockdown of Wnt7b inhibited OD of hASCs.

\section{Role of $\beta$-catenin on hASCs OD induced by cyclic strain}

For determining effects of transfection, $\beta$-catenin expressions of hASCs, which were transfected with the EX- $\beta$ catenin, si $\beta$-catenin, EX-Ctrl, and siR-Ctrl, were examined by RT-PCR, western blotting, and immunofluorescence. The results of RT-PCR analysis indicated that the $\beta$ catenin expression in EX- $\beta$-catenin group showed an increase of $2.51 \pm 0.69-\mathrm{F}(p=0.001)$ in comparison to the EX-Ctrl group; the $\beta$-catenin expression in the si $\beta$-catenin group significantly reduced $3.15 \pm 0.89-\mathrm{F}(p=0.002)$ in comparison to the siR-Ctrl group (Fig. 6a). The results of western blot analysis indicated that the cytoplasmic and nuclear $\beta$-catenin expression in the EX- $\beta$-catenin group showed an increase of $1.40 \pm 0.07-\mathrm{F}(p=0.004)$ and $1.42 \pm$ $0.04-\mathrm{F}(p=0.002)$ in comparison to the EX-Ctrl group; the cytoplasmic $\beta$-catenin and nuclear $\beta$-catenin expression in the si $\beta$-catenin group significantly reduced $1.38 \pm 0.12-\mathrm{F}$ $(p=0.002)$ and $1.38 \pm 0.09-\mathrm{F}(p=0.032)$ in comparison to the siR-Ctrl group (Fig. 6b).

Furthermore, RT-PCR analysis was used to determine effects of $\beta$-catenin on the OD of hASCs after loading cyclic strain for 6 days. After EX- $\beta$-catenin transfection, the results showed that $\mathrm{R}, \mathrm{A}, \mathrm{S}, \mathrm{W} 2, \mathrm{~W} 7, \beta$ significantly enhanced $3.60 \pm 1.07-\mathrm{F} \quad(p=0.022), \quad 3.21 \pm 0.49-\mathrm{F} \quad(p=$ $0.002), 2.45 \pm 0.29-\mathrm{F}(p=0.004), 4.30 \pm 1.12-\mathrm{F}(p=0.001)$, $4.63 \pm 1.88$-F $(p=0.006)$, and $5.17 \pm 1.04-\mathrm{F} \quad(p=0.002)$, respectively, in comparison to the EX-Ctrl group (Fig. 6c). After si $\beta$-catenin transfected, the results showed that R, A, S, W2, W7, $\beta$ significantly decreased $4.92 \pm 1.46-\mathrm{F}$ 


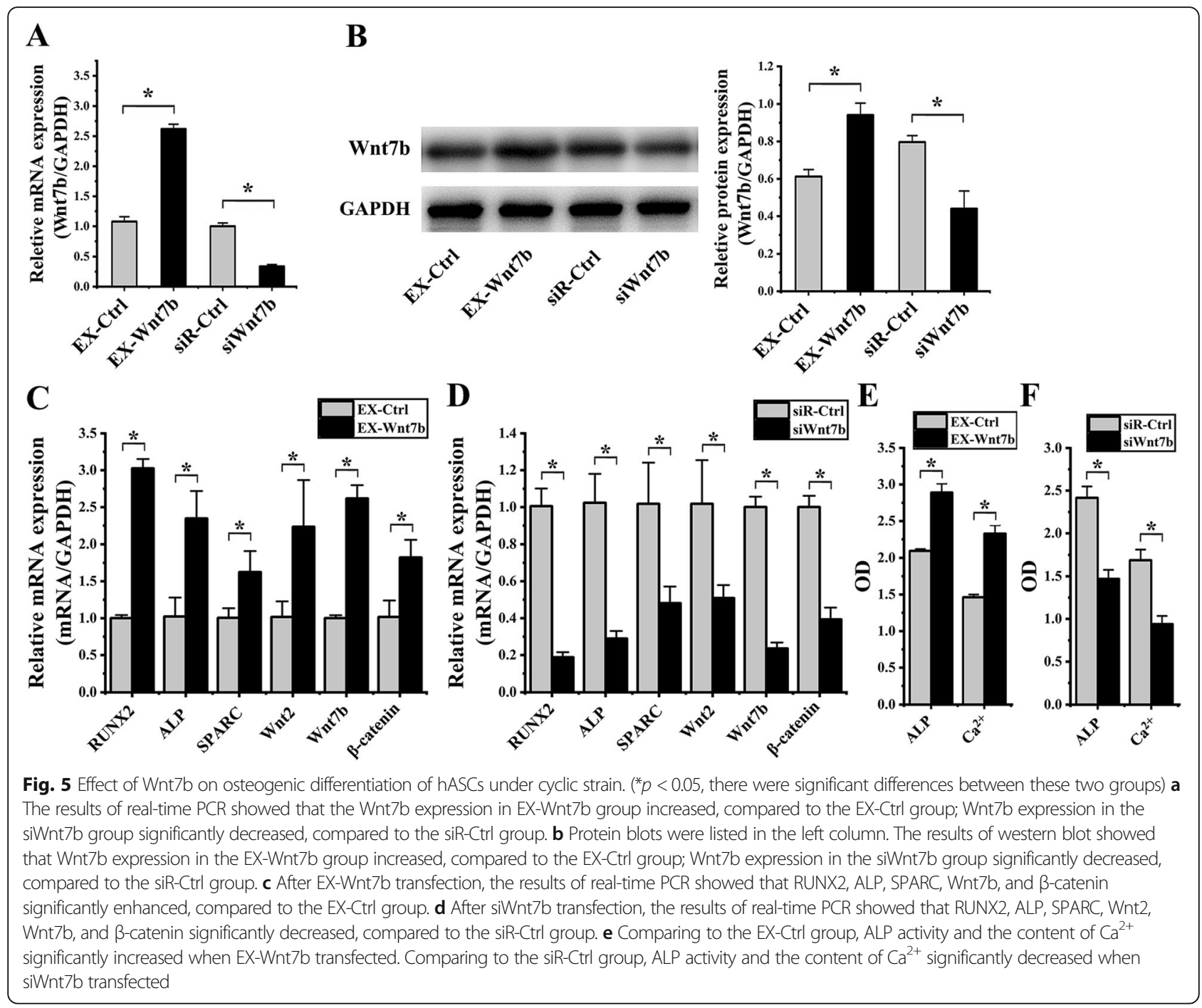

$(p=0.001), \quad 3.41 \pm 0.38-\mathrm{F} \quad(p<0.001), 2.66 \pm 1.27-\mathrm{F} \quad(p=$ $0.010), 2.23 \pm 0.33-\mathrm{F}(p=0.004), 3.17 \pm 1.27-\mathrm{F}(p=0.005)$ and $3.38 \pm 0.10-\mathrm{F}(p=0.003)$ respectively, compared to the siR-Ctrl group (Fig. 6d). After EX- $\beta$-catenin transfection, ALP activity and the content of $\mathrm{Ca}^{2+}$ significantly increased $1.60 \pm 0.16-\mathrm{F}(p<0.001)$ and $1.37 \pm 0.12-\mathrm{F}(p=$ 0.003), respectively, in comparison to the EX-Ctrl group (Fig. 6e). After siß-catenin transfected, ALP activity and the content of $\mathrm{Ca} 2+$ significantly decreased $1.50 \pm 0.19-\mathrm{F}$ $(p=0.021)$ and $1.67 \pm 0.06-\mathrm{F}(p<0.001)$, respectively, compared to the siR-Ctrl group (Fig. 6f).

All these results above suggested that overexpression of $\beta$-catenin enhanced OD of hASCs, and knockdown of $\beta$-catenin inhibited OD of hASCs.

Role of miR-503-3p overexpression and inhibition on hASCs OD induced by cyclic strain

For determining efficiency of transfection with miR-503$3 \mathrm{p}$ mimic and inhibitor, miR-503-3p expressions in
hASCs were detected by RT-PCR and western blot. After transfecting miR-503-3p mimic in hASCs, miR503-3p markedly increased $411.49 \pm 82.72-\mathrm{F}(p<0.001)$ than the group of MCM (Fig. 7a). After transfecting miR-503-3p inhibitor in hASCs, the expression of miR503 -3p reduced $2.43 \pm 0.02-\mathrm{F}(p=0.006)$ than the group of miR-Ctrl inhibitor (Fig. 7b).

Furthermore, the role of miR-503-3p on hASCs OD was determined by RT-PCR, western blotting, colorimetric assay, and immunofluorescence. Compared to the MCM group, RT-PCR analysis was used to detect mRNA expression levels in hASCs transfected with miR503-3p mimic, results indicated that $\mathrm{R}, \mathrm{A}, \mathrm{S}, \mathrm{W} 2, \mathrm{~W} 7, \beta$ showed a decrease of $1.90 \pm 0.15-\mathrm{F}(p=0.028), 2.20 \pm$ $0.02-\mathrm{F}(p=0.001), 1.34 \pm 0.08-\mathrm{F}(p=0.004), 3.17 \pm 1.10-\mathrm{F}$ $(p=0.005), \quad 1.44 \pm 0.20-\mathrm{F}(p=0.018)$, and $4.23 \pm 0.50-\mathrm{F}$ $(p=0.001)$, respectively (Fig. $7 \mathrm{c}$ ); proteins of $\mathrm{R}, \mathrm{A}, \mathrm{S}$, W2, W7, $\beta$ cytoplasmic and nuclear detected by western blot reduced $1.90 \pm 0.97-\mathrm{F}(p=0.030), 2.37 \pm 0.38-\mathrm{F}$ ( $p=$ 


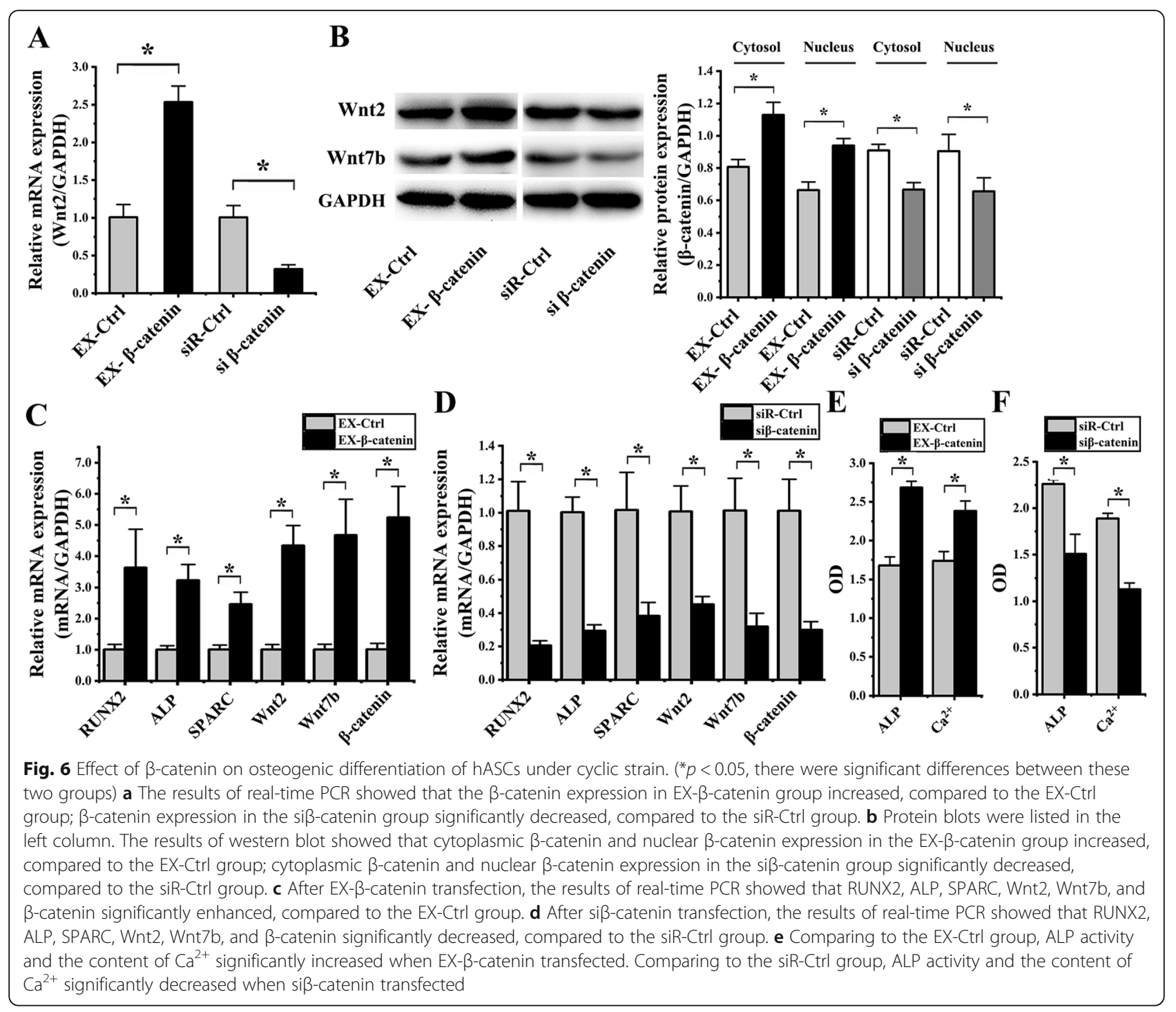

$0.001), 1.57 \pm 0.24-\mathrm{F}(p=0.022), 1.43 \pm 0.15-\mathrm{F}(p=0.038)$, $1.55 \pm 0.16-\mathrm{F} \quad(p=0.007), 1.45 \pm 0.25-\mathrm{F} \quad(p=0.014)$, and $1.71 \pm 0.26-\mathrm{F}(p=0.007)$, respectively (Fig. $7 \mathrm{~d})$; ALP activity and the content of $\mathrm{Ca}^{2+}$ significantly decreased $1.43 \pm 0.14-\mathrm{F}(p=0.003)$ and $1.93 \pm 0.16-\mathrm{F}(p=0.011)$, respectively (Fig. 7e); $\beta$-catenin expression in cytoplasmic and nucleus showed a decrease in hASCs transfected with the miR-503-3p mimic detected by immunofluorescence (Fig. 7f).

Compared to the miR-Ctrl inhibitor group, RT-PCR analysis was used to detect the following mRNA expression levels in hASCs that had undergone transfection with miR-503-3p inhibitor, results indicated that $\mathrm{R}, \mathrm{A}, \mathrm{S}$, W2, W7, $\beta$ showed an increase of $2.97 \pm 0.11-\mathrm{F}(p<$ $0.001), 4.38 \pm 1.17-\mathrm{F}(p<0.001), 2.22 \pm 0.53-\mathrm{F}(p=0.001)$, $2.10 \pm 0.44-\mathrm{F} \quad(p=0.006), 2.45 \pm 0.30-\mathrm{F} \quad(p<0.001)$, and $1.91 \pm 0.24-\mathrm{F}(p=0.021)$ respectively (Fig. $7 \mathrm{~g})$; the protein expression of above markers detected by western blot reduced $1.73 \pm 0.13-\mathrm{F}(p=0.023), 1.69 \pm 0.04-\mathrm{F}(p=$ $0.004), 2.09 \pm 0.09-\mathrm{F}(p=0.001), 1.43 \pm 0.07-\mathrm{F}(p=0.006)$, $1.46 \pm 0.34-\mathrm{F} \quad(p=0.009), 1.35 \pm 0.06-\mathrm{F} \quad(p=0.002)$, and $1.70 \pm 0.29-\mathrm{F}(p=0.008)$, respectively (Fig. 7h); ALP activity and the content of $\mathrm{Ca}^{2+}$ significantly increased $2.05 \pm 0.05-\mathrm{F}(p<0.001)$ and $1.60 \pm 0.30-\mathrm{F}(p=0.004)$, respectively (Fig. 7i); $\beta$-catenin expression in cytoplasmic and nucleus were increased in hASCs transfected with miR-503-3p mimic detected by immunofluorescence (Fig. 7j).

Our findings suggested that the overexpression of miR-503-3p inhibited OD of hASCs, while the suppression of miR-503-3p promoted its OD of hASCs.

Roles of Wnt2 and Wnt7b regulated by miR-503-3p on hASCs OD induced by cyclic strain

Real-time PCR, western blotting, colorimetric assay, and immunofluorescence were utilized to investigate the 


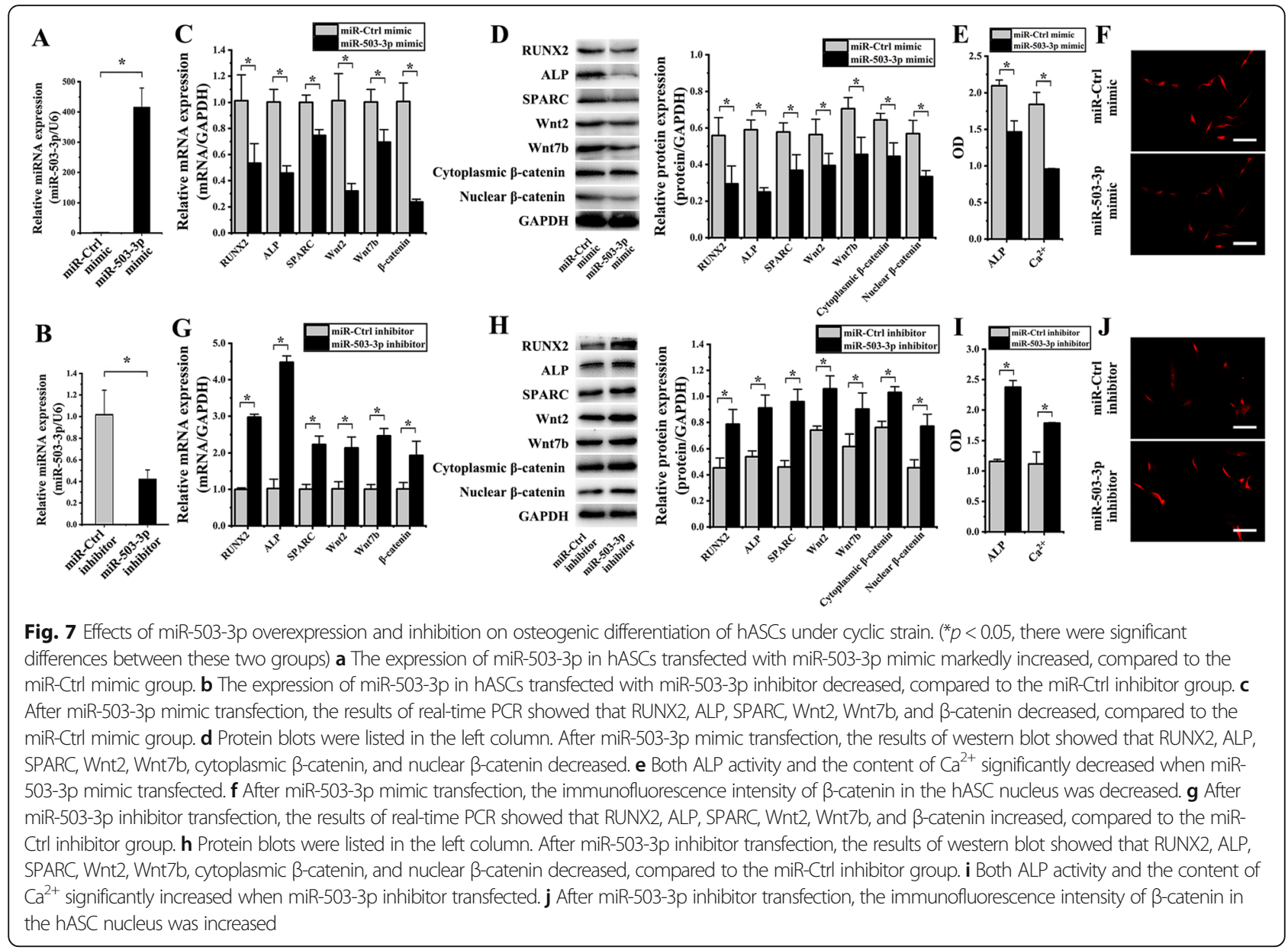

molecular mechanisms of miR-503-3p on OD through regulating Wnt2 and Wnt7b expression in hASCs induced by cyclic strain.

After co-transfecting miR-503-3p mimic with EX-Wnt2 and EX-Wnt7b into hASCs, the following mRNA expression levels were detected by RT-PCR analysis, results showed that R, A, S, W2, W7, $\beta$ showed an increase of $2.67 \pm 1.28-\mathrm{F}(p=0.003), 2.43 \pm 0.35-\mathrm{F}(p=0.005), 1.73 \pm$ $0.19-\mathrm{F}(p=0.010), 2.06 \pm 0.30-\mathrm{F}(p=0.006), 2.15 \pm 1.33-\mathrm{F}$ $(p=0.016)$, and $2.59 \pm 0.30-\mathrm{F}(p=0.011)$, respectively, than the group of miR-503-3p mimic and EX-Ctrl (Fig. 8a); protein expression of R, A, S, W2, W7, $\beta$ cytoplasmic and nuclear also showed an increase of $1.54 \pm 0.11-\mathrm{F}(p=$ $0.001), 1.44 \pm 0.14-\mathrm{F}(p=0.002), 1.32 \pm 0.03-\mathrm{F}(p=0.006)$, $1.34 \pm 0.04-\mathrm{F}(p=0.036), 2.96 \pm 0.35-\mathrm{F}(p=0.001), 1.77 \pm$ $0.22-\mathrm{F}(p=0.001)$, and $1.59 \pm 0.14-\mathrm{F}(p=0.010)$, respectively, than the group of miR-503-3p mimic and EX-Ctrl (Fig. 8b); ALP activity and the content of $\mathrm{Ca}^{2+}$ significantly increased $1.68 \pm 0.04-\mathrm{F}(p<0.001)$ and $2.61 \pm 0.01-\mathrm{F}(p<$ 0.001 ), respectively (Fig. 8c); $\beta$-catenin expression in cytoplasmic and nucleus were increased in hASCs that had undergone transfection with the EX-Wnt2 and EX-Wnt7b detected by immunofluorescence (Fig. 8d). The above results suggested that Wnt2 and Wnt7b overexpression could remedy the inhibiting effect of miR-503-3p mimic on hASCs OD induced by cyclic strain.

After co-transfecting miR-503-3p inhibitor with siWnt2 and siWnt7b into hASCs, the following mRNA expression levels were detected by RT-PCR analysis, results showed that $\mathrm{R}, \mathrm{A}, \mathrm{S}, \mathrm{W} 2, \mathrm{~W} 7, \beta$ reduced $1.96 \pm 0.49-\mathrm{F}(p=0.004)$, $4.17 \pm 1.79-\mathrm{F}$ ( $p=0.013), 2.12 \pm 0.52-\mathrm{F}(p=0.003), 3.20 \pm$ $1.22-\mathrm{F}(p=0.002), 2.41 \pm 0.23-\mathrm{F}(p=0.002)$, and $2.60 \pm$ $1.03-\mathrm{F}(p=0.008)$, respectively, than the group of miR503-3p inhibitor and siR-Ctrl (Fig. 8e); proteins of R, A, S, W2, W7, $\beta$ cytoplasmic and nuclear $\beta$-catenin also showed a decrease of $1.22 \pm 0.15-\mathrm{F}(p=0.044), 2.12 \pm 0.58-\mathrm{F}(p=$ $0.003), 1.48 \pm 0.15-\mathrm{F}(p=0.005), 1.31 \pm 0.05-\mathrm{F}(p=0.009)$, $1.60 \pm 0.23-\mathrm{F} \quad(p=0.035), 1.32 \pm 0.07-\mathrm{F} \quad(p=0.015)$, and $1.36 \pm 0.12-\mathrm{F}(p=0.010)$, respectively, than the group of miR-503-3p inhibitor and siR-Ctrl (Fig. 8f); ALP activity and the content of $\mathrm{Ca}^{2+}$ significantly decreased $1.39 \pm$ $0.16-\mathrm{F}(p=0.002)$ and $2.10 \pm 0.09-\mathrm{F}(p<0.001)$, respectively (Fig. 8g); the expression of $\beta$-catenin in cytoplasmic and nucleus were decreased in hASCs transfected with siWnt2 and siWnt7b detected by immunofluorescence (Fig. 8h). The above results suggested that Wnt2 and 


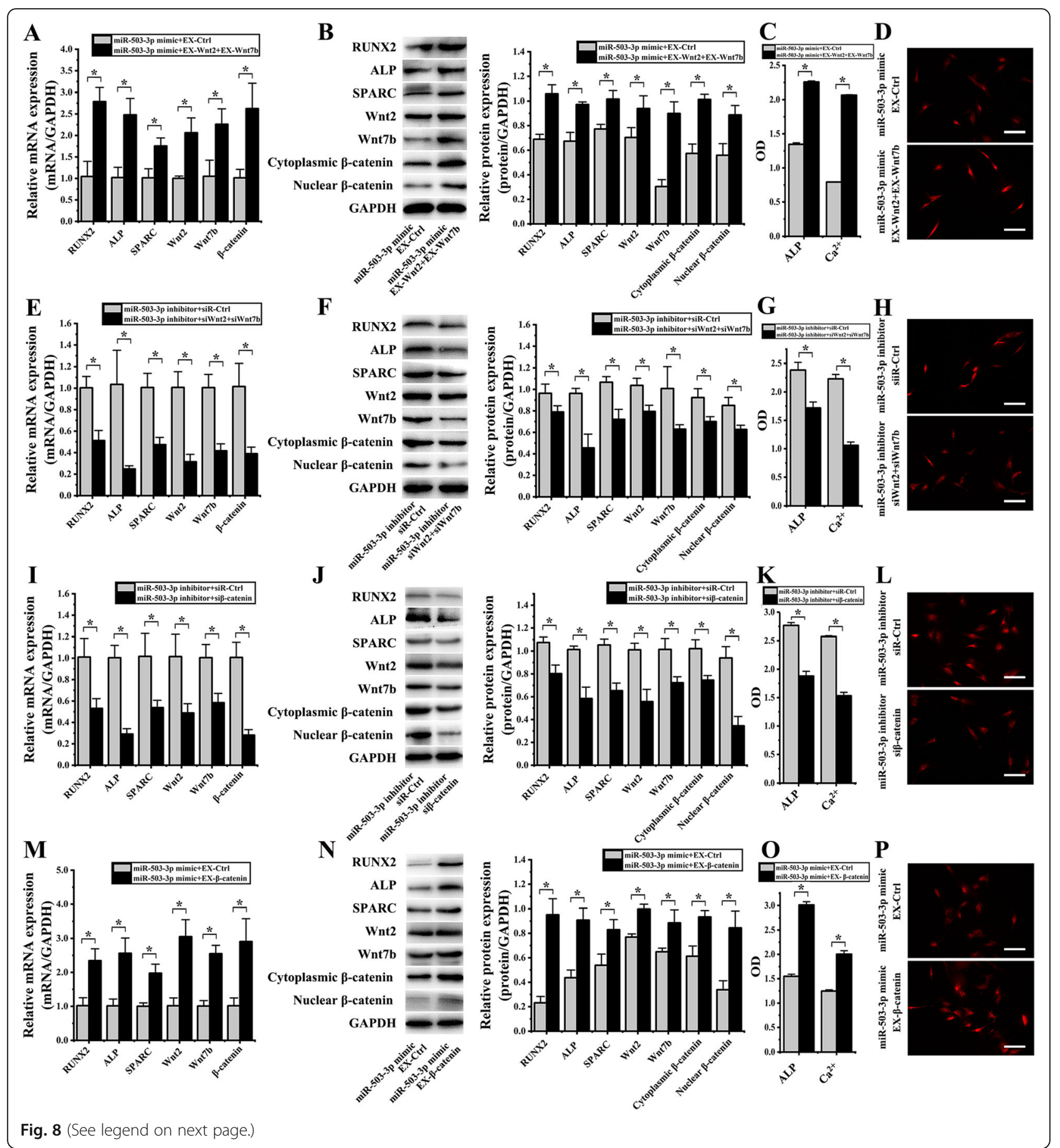

Wnt7b downregulation could weaken the potentiation of miR-503-3p inhibitor on OD of hASCs under cyclic strain.

After co-transfecting miR-503-3p inhibitor with si $\beta$ catenin into hASCs, the following mRNA expression levels were detected by RT-PCR analysis, results indicated that $\mathrm{R}, \mathrm{A}, \mathrm{S}$ decreased $1.90 \pm 0.60-\mathrm{F}(p=0.013), 3.45 \pm$ $0.90-\mathrm{F}(p=0.001)$, and $1.89 \pm 0.56-\mathrm{F}(p=0.021)$ respectively, and W2, W7, $\beta$ decreased $2.08 \pm 0.70-\mathrm{F}(p=0.015)$,
$1.72 \pm 0.16-\mathrm{F}(p=0.008)$, and $3.58 \pm 0.47-\mathrm{F}(p=0.001)$ respectively, than the group of miR-503-3p inhibitor and siR-Ctrl (Fig. 8i); proteins of R, A, S decreased $1.34 \pm 0.17$ $\mathrm{F}(p=0.005), 1.73 \pm 0.39-\mathrm{F}(p=0.003)$, and $1.61 \pm 0.37-\mathrm{F}$ $(p=0.014)$, respectively, proteins of Wnt2, Wnt7b, cytoplasmic and nuclear $\beta$-catenin showed a decrease of $1.81 \pm 0.31-\mathrm{F}(p=0.002), 1.40 \pm 0.09-\mathrm{F}(p=0.001), 1.37 \pm$ $0.15-\mathrm{F}(p=0.005)$, and $2.72 \pm 0.55-\mathrm{F}(p=0.001)$, respectively, than the group of miR-503-3p inhibitor and siR-Ctrl 
(See figure on previous page.)

Fig. 8 Effect of Wnt2 and Wnt7b regulated by miR-503-3p on osteogenic differentiation of hASCs under cyclic strain $\left({ }^{*} p<0.05\right.$, there were significant differences between these two groups) a After co-transfecting miR-503-3p mimic with EX-Wnt2 and EX-Wnt7b, real-time PCR showed RUNX2, ALP, SPARC, Wnt2, Wnt7b, and $\beta$-catenin were increased, than the miR-503-3p mimic and EX-Ctrl group. $\mathbf{b}$ Protein blots were listed in the left column. After cotransfecting miR-503-3p mimic with EX-Wnt2 and EX-Wnt7b, western blot showed that RUNX2, ALP, SPARC, Wnt2, Wnt7b, cytoplasmic, and nuclear $\beta$ catenin increased, than the miR-503-3p mimic and EX-Ctrl group. c Both ALP activity and the content of $\mathrm{Ca}^{2+}$ significantly increased when co-transfecting miR-503-3p mimic with EX-Wnt2 and EX-Wnt7b. d After co-transfecting miR-503-3p mimic with EX-Wnt2 and EX-Wnt7b, the immunofluorescence intensity of nucleus $\beta$-catenin was enhanced. e After co-transfecting miR-503-3p inhibitor with siWnt2 and siWnt7b, real-time PCR showed that RUNX2, ALP, SPARC, Wnt2, Wnt7b, and $\beta$-catenin decreased, than the miR-503-3p inhibitor and siR-Ctrl group. $\mathbf{f}$ Protein blots were listed in the left column. After co-transfecting miR-503-3p inhibitor with siWnt2 and siWnt7b, western blot showed RUNX2, ALP, SPARC, Wnt2, Wnt7b, cytoplasmic, and nuclear $\beta$-catenin decreased, than the miR-503-3p inhibitor and siR-Ctrl group. $\mathbf{g}$ Both ALP activity and the content of $\mathrm{Ca}^{2+}$ significantly decreased when co-transfecting miR-503-3p inhibitor with siWnt2 and siWnt7b. $\mathbf{h}$ After co-transfecting miR-503-3p inhibitor with siWnt2 and siWnt7b, the immunofluorescence intensity of nucleus $\beta$-catenin was decreased. i After co-transfecting miR-503-3p inhibitor with si $\beta$-catenin, real-time PCR showed that RUNX2, ALP, and SPARC decreased; Wnt2, Wnt7b and $\beta$ catenin decreased, than the miR-503-3p inhibitor and siR-Ctrl group. $\mathbf{j}$ Protein blots were listed in the left column. After co-transfecting miR-503-3p inhibitor with siß-catenin, western blot showed that RUNX2, ALP, and SPARC decreased; Wnt2, Wnt7b, cytoplasmic and nuclear $\beta$-catenin decreased, than the miR503-3p inhibitor and siR-Ctrl group. $\mathbf{k}$ Both ALP activity and the content of $\mathrm{Ca}^{2+}$ significantly decreased when co-transfecting miR-503-3p inhibitor with si 3 catenin. I After co-transfecting miR-503-3p inhibitor with siß-catenin into hASCs, the immunofluorescence intensity of nucleus $\beta$-catenin was decreased. $\mathbf{m}$ After co-transfecting miR-503-3p mimic with EX- $\beta$-catenin, real-time PCR showed that RUNX2, ALP, and SPARC increased; Wnt2, Wnt7b, and $\beta$-catenin increased, than the miR-503-3p mimic and EX-Ctrl group. $\mathbf{n}$ Protein blots were listed in the left column. After co-transfecting miR-503-3p mimic with EX- $\beta$ catenin, western blot showed that RUNX2, ALP, and SPARC increased; Wnt2, Wnt7b, cytoplasmic, and nuclear $\beta$-catenin increased, than the miR-503-3p mimic and EX-Ctrl group. o Both ALP activity and the content of $\mathrm{Ca}^{2+}$ significantly increased when co-transfecting miR-503-3p mimic with EX- $\beta$-catenin. $\mathbf{p}$ After co-transfecting miR-503-3p mimic with EX- $\beta$-catenin, the immunofluorescence intensity of nucleus $\beta$-catenin was increased

(Fig. 8j); ALP activity and the content of $\mathrm{Ca}^{2+}$ significantly decreased $1.94 \pm 0.02-\mathrm{F}(p<0.001)$ and $1.61 \pm 0.06-\mathrm{F}(p<$ 0.001 ), respectively (Fig. $8 \mathrm{k}$ ); the expression of $\beta$-catenin in cytoplasmic and nucleus were decreased in hASCs cotransfected miR-503-3p inhibitor with si $\beta$-catenin detected by immunofluorescence (Fig. 8l).

After co-transfecting miR-503-3p mimic with EX- $\beta$ catenin into hASCs, mRNA expression levels were detected by RT-PCR analysis. Results showed that R, A, S showed an increase of $2.30 \pm 0.97-\mathrm{F}(p=0.006), 2.52 \pm$ $0.40-\mathrm{F}(p=0.006)$, and $1.97 \pm 0.46-\mathrm{F}(p=0.004)$ respectively, and W2, W7, $\beta$ increased $3.00 \pm 0.21-\mathrm{F}(p=0.003)$, $2.53 \pm 0.26-\mathrm{F}(p=0.001)$, and $2.86 \pm 0.82-\mathrm{F}(p=0.010)$, respectively, than the group of miR-503-3p mimic and EXCtrl (Fig. 8m); proteins of R, A, S also increased $4.10 \pm$ $1.05-\mathrm{F}(p=0.001), 2.08 \pm 0.33-\mathrm{F}(p=0.002)$, and $1.54 \pm$ 0.08 -F ( $p=0.032$ ), respectively, proteins of Wnt2, Wnt7b, cytoplasmic and nuclear $\beta$-catenin showed an increase of $1.30 \pm 0.03-\mathrm{F}(p=0.001), 1.36 \pm 0.12-\mathrm{F}(p=0.022), 1.52 \pm$ $0.12-\mathrm{F}(p=0.007)$, and $2.49 \pm 0.20-\mathrm{F}(p=0.015)$, respectively, than the group of miR-503-3p mimic and EX-Ctrl (Fig. 8n); ALP activity and the content of $\mathrm{Ca}^{2+}$ significantly increased $1.47 \pm 0.04-\mathrm{F}(p<0.001)$ and $1.68 \pm 0.02-\mathrm{F}(p=$ 0.001 ), respectively (Fig. 8o); the expression of $\beta$-catenin in cytoplasmic and nucleus were increased in hASCs that had undergone co-transfection with the miR-503-3p mimic and EX- $\beta$-catenin detected by immunofluorescence (Fig. 8p).

\section{Discussion}

Although there are many advantages of hASCs mentioned in the "Introduction" section for construction of tissue engineering, the osteogenic ability of ASCs is lower than bone mesenchymal stem cells (BMSCs). Shafiee et al. claimed that ASCs had reduced ALP activity and mineralization when compared to BMSCs during OD on the 7th and 14th day [19]. The potent osteogenic capacity of BMSCs in comparison to ASCs was also proved by Vishnubalaji et al. by the following experiments: calcium mineralization, cytochemical qualitative analysis, and RTPCR of osteocalcin, ALP, and osteopontin [20]. Park et al. concluded the human mesenchymal stem cells were more sensitive to mechanical stimulation and more effective toward OD than the hASCs under these modes of mechanical stimulation [21]. Even so, hASCs is still one of the seed cells that can be chosen for construction of tissue engineering bone.

All tissues survive in a mechanical environment, which plays an essential role in maintaining biological activities [22-24]. In stem cells, common biomechanical stimuli that induce osteogenesis are tension, compression, and fluid shear stress. Under 5\% and below strain, ALP activity and RUNX2 gene expression was found to increase in mouse bone marrow stromal cells but, on the other hand, showed a reduction with higher strains [25]. This result showed that OD is promoted by low tension levels while high levels of tension inhibit that. Compression has a role in chondrogenic as well as OD in human BMSCs. Jagodzinski et al. claimed that mesenchymal stem cells were applied to $10 \%$ cyclic compression with continuous perfusion; then, the expression of Runx2 and osteocalcin was increased [26]. Li et al. reported that the proliferation rates of human mesenchymal stem cells increased after loading fluid flow for $24 \mathrm{~h}$, and gene expression of osteocalcin and osteopontin showed an increase [27]. In addition to providing support for the construction of tissue engineered bone tissue in vitro, these findings can also provide a theoretical basis for stem cells therapy to repair bone defects in vivo. 
Various studies confirmed that Wnt2 was an extracellular activator of the W- $\beta$ signaling pathway [28]. Some studies indicated that Wnt2 is closely related to osteogenesis. The mRNA levels of Wnt2 were higher in osteoblasts compared to their progenitors [29]. When compared to adjacent non-cancerous tissues, the expression of Wnt2 protein was elevated in human osteosarcoma tissues. It was also seen that there was a marked increase in expression in MG63 OS cell line in comparison with the human osteoblast hFOB 1.19 cell line [30]. Wnt2 in human dental follicle cells were significantly upregulated after induction by human dental follicle cells conditioned medium compared to human dental follicle cells in normal medium [31].

Some studies confirmed that Wnt2 were direct regulated by some microRNAs for controlling biological activities. miR-199a-5p has the ability to regulate myogenesis by suppressing Wnt signaling factors that play a role in balancing myogenic cell proliferation and differentiation by targeting FZD4, JAG1, and Wnt2 [32]. In the smooth muscle, MiR-199a-5p, which targets Wnt2, has a vital role in Wnt2-mediated regulation of proliferative and differentiation processes. It might act as a critical modulator of smooth muscle hypertrophy, which is vital for organ remodeling [33]. Promotion of proliferation of esophageal squamous cell carcinoma cell is brought about when miR-30a-3p/5p is regulated. This is brought about when Wnt2 and Fzd2 get inhibited, thereby activating the Wnt signaling pathway [34]. Furthermore, ectopic expression of miR-30a-3p significantly suppressed the migration, proliferation, and invasion of a human RCC cell line in vitro, while miR-30a-3p could inhibit tumor growth in vivo as well. Overexpression of miR-199a/b-5p, then inhibiting Wnt2, reduced autophagy, and induced cell apoptosis result in enhanced imatinib's efficacy in K562R cells [35].

Various studies confirmed that Wnt7b was an extracellular activator and activator of the $W-\beta$ signaling pathway [36]. Overexpression of Wnt7b in 1-month-old mice for 1 week markedly stimulated bone formation [37]. Out of the 19 Wnt ligands, it was found that Wnt7b was the most load-responsive during the formation of bone in aging C57Bl/6JN mice [38]. Wnt7b not only promotes bone formation through the W- $\beta$ pathway but also in part through mTORC1 activation [39].

Some studies confirmed that some kinds of microRNA target Wnt7b for controlling biological activities. In human aortic smooth muscle cells, miR-29b mimic could target Wnt7b and potently repress $\mathrm{Wnt7b} / \beta$-catenin protein expression, whereas miR-29b anti-miR had the ability to increase their expression. This indicated that miR-29b brings about negative regulation of $W n t 7 b / \beta-$ catenin signaling [40]. Overexpression of miR-G-1 inhibited the expression of Wnt7b, and then inhibiting cell proliferation, cell cycle progression, migration, invasion, and drug resistance in cervical cancer cells [41].

Some studies have proofed the miR-503-3p expression changed in many types of ocological lesions. It regulates p21 and CDK4 expression thereby inducing apoptosis of lung cancer cells [42]. miRNA expression profiles of lymphatic endothelial cells and functional analysis indicate that miR-503-3p might be as downstream targets of ELK3 in lymphatic endothelial cells, which cause to promote the migrating and invasive ability of breast cancer cells such as MDA-MB-231, Hs578T, and BT20 in vitro [43]. miR-503-3p promotes epithelial-mesenchymal transition in breast cancer by directly targeting SMAD2 and E-cadherin [44]. miR-503-3p inhibited tumor growth via the regulation of cancer stem cell proliferation and selfrenewal, and it may function as a stemness-attenuating factor via cell-to-cell communications [45]. It was upregulated in plasma from primary resistance patients of epidermal growth factor receptor tyrosine kinase inhibitors in patients of non-small cell lung cancer [46]. miR-503$3 p$ also play a critical role in non-oncological disease. miR-503-3p were differentially expressed between diabetic kidney disease cases and type 1 diabetes mellitus patients controls [47]. miR-503-3p was significantly downregulated in rats with acute respiratory distress syndrome and acute lung injury, who acquired the treatment of bone marrow-derived mesenchymal stem cells [48]. The function of miR-503-3p in bone metabolism is still unknown. Our study is the first to verify a novel role and target of miR-503-3p during OD. It has the potential to become a new regulated target for bone regeneration. We hypothesize that the inhibition of miR-503-3p could promote the osteogenic differentiation of hASCs during the construction of tissue-engineered bone in vitro, shorten the time, and accelerate the bone deposition.

\section{Conclusion}

In this study, we designed various experiments to examine the function of miR-503-3p during the process of hASCs OD induced by cyclic strain. Cyclic strain regulated the process of hASCs OD in vitro by downregulating the expression of miR-503-3p and upregulating the expression of Wnt 2 and Wnt7b. Furthermore, by modulating miR-503-3p activity, we conclude that miR-503-3p inhibit the W- $\beta$ pathway by targeting Wnt2 and Wnt7b, which then inhibit the OD of hASCs induced by cyclic strain in vitro.

\section{Abbreviations}

miRNAs: MicroRNAs; OD: Osteogenic differentiation; hASCs: Human adiposederived stem cells; W- $\beta$ : Wnt/ $\beta$-catenin; siRNA: Small interfering RNA; RTPCR: Quantitative real-time PCR; UTR: Untranslated region; RUNX2: Runtrelated transcription factor 2; a-MEM: Alpha-modified Eagle's medium; FBS: Fetal bovine serum; EX-Wnt2: Wnt2 expression plasmid; siWnt2: Wnt2 siRNA; EX-Wnt7b: Wnt7b expression plasmid; siWnt7b: Wnt7b siRNA; EXCtrl: Negative control expression plasmid; siR-Ctrl: Negative control siRNA; 
miR-Ctrl mimic: Mimic negative control of the miR-503-3p mimic; miR-Ctrl inhibitor: Inhibitor negative control of the miR-503-3p inhibitor; wtWnt2: Wnt2 3'UTR-wild type; mu-Wnt2: Wnt2 3'UTR-mutant; wtWnt7b: Wnt7b 3'UTR-wild type; mu-Wnt7b: Wnt7b 3'UTR-mutant; si catenin: Short interfering RNAs that targeted $\beta$-catenin; ALP: Alkaline phosphatase; SPARC: Secreted protein acidic and cysteine-rich; GAPD $\mathrm{H}$ : Glyceraldehyde-3-phosphate dehydrogenase; TBS: Tris-buffer saline; F: Fold; MCM: miR-Ctrl mimic; R: RUNX2; A: ALP; S: SPARC; W7: Wnt7b; $\beta$ : $\beta$ Catenin; BMSCs: Bone marrow mesenchymal stem cells

\section{Acknowledgements}

All authors are acknowledged for their contribution to the study.

\section{Authors' contributions}

Hongming Du designed this experiment. Yadong Luo, Xu Ding, Huan Ji, Meng Li, and Haiyang Song performed all procedures of experiments. Hongming Du supervised the project and cooperated with Sheng Li and Chenxing Wang to analyze data. Hongming Du and Heming Wu wrote the manuscript. All authors edited and approved the manuscript.

\section{Authors' information}

Not applicable.

\section{Funding}

This study was supported by the "National Natural Science Foundation of China (No. 81600908)" and "Technology Development Fund of Nanjing Medical University (2017NJMU103)".

\section{Availability of data and materials}

The datasets generated and/or analyzed during the current study are not publicly available but are available from the corresponding author upon reasonable request.

\section{Ethics approval and consent to participate}

This study was approved by the ethical committee of the Nanjing Medical University. All cells donors signed informed consent documents. The authors declare that they have no conflict of interest.

\section{Consent for publication}

Not applicable

\section{Competing interests}

The authors have no competing interests to declare.

Received: 20 February 2020 Revised: 5 July 2020

Accepted: 20 July 2020 Published online: 25 July 2020

\section{References}

1. Zuk PA, Zhu M, Ashjian P, et al. Human adipose tissue is a source of multipotent stem cells. Mol Biol Cell. 2002;13(12):4279-95.

2. Chen S, Zheng Y, Zhang S, et al. Promotion effects of miR-375 on the osteogenic differentiation of human adipose-derived mesenchymal stem cells. Stem Cell Reports. 2017;8(3):773-86.

3. Gentile P, Piccinno MS, Calabrese C. Characteristics and potentiality of human adipose-derived stem cells (hASCs) obtained from enzymatic digestion of fat graft. Cells. 2019;8(3):E282.

4. Huang SJ, Fu RH, Shyu WC, et al. Adipose-derived stem cells: isolation, characterization, and differentiation potential. Cell Transplant. 2013;22(4): 701-9.

5. Vidal MA, Walker NJ, Napoli E, et al. Evaluation of senescence in mesenchymal stem cells isolated from equine bone marrow, adipose tissue, and umbilical cord tissue. Stem Cells Dev. 2012;21(2):273-83.

6. Du HM, Zheng $X H$, Wang $L Y$, et al. The osteogenic response of undifferentiated human adipose-derived stem cells under mechanical stimulation. Cells Tissues Organs. 2012;196(4):313-24.

7. Hanson AD, Marvel SW, Bernacki SH, et al. Osteogenic effects of rest inserted and continuous cyclic tensile strain on hASC lines with disparate osteodifferentiation capabilities. Ann Biomed Eng. 2009;37(5):955-65.

8. Maredziak M, Lewandowski D, Tomaszewski KA, et al. The effect of lowmagnitude low-frequency vibrations (LMLF) on osteogenic differentiation potential of human adipose derived mesenchymal stem cells. Cell Mol Bioeng. 2017:10(6):549-62.

9. Virjula S, Zhao F, Leivo J, et al. The effect of equiaxial stretching on the osteogenic differentiation and mechanical properties of human adipose stem cells. J Mech Behav Biomed Mater. 2017;72:38-48.

10. Houschyar KS, Tapking C, Borrelli MR, et al. Wnt pathway in bone repair and regeneration - what do we know so far. Front Cell Dev Biol. 2018;6:170.

11. MacDonald BT, Tamai K, He X. Wnt/beta-catenin signaling: components, mechanisms, and diseases. Dev Cell. 2009;17(1):9-26.

12. Chen $\mathrm{H}, \mathrm{Mo} \mathrm{D}$, Li M, et al. miR-709 inhibits 3T3-L1 cell differentiation by targeting GSK3beta of Wnt/beta-catenin signaling. Cell Signal. 2014;26(11): 2583-9.

13. Riehl BD, Park JH, Kwon IK, et al. Mechanical stretching for tissue engineering: two-dimensional and three-dimensional constructs. Tissue Eng Part B Rev. 2012;18(4):288-300.

14. Pan JX, Xiong L, Zhao K, et al. YAP promotes osteogenesis and suppresses adipogenic differentiation by regulating beta-catenin signaling. Bone Res. 2018;6(2):18.

15. Bartel DP. MicroRNAs: target recognition and regulatory functions. Cell. 2009;136(1):215-33.

16. Zeng $Z \mathrm{~L}$, Lin $\mathrm{XL}$, Tan $\mathrm{LL}$, et al. MicroRNAs: important regulators of induced pluripotent stem cell generation and differentiation. Stem Cell Rev Rep. 2018;14(1):71-81.

17. Li N, Long B, Han W, et al. microRNAs: important regulators of stem cells. Stem Cell Res Ther. 2017:8(1):110.

18. Luo Y, Ge R, Wu H, Ding X, Song H, Ji H, et al. The osteogenic differentiation of human adipose-derived stem cells is regulated through the let-7i-3p/LEF1/ $\beta$-catenin axis under cyclic strain. Stem Cell Res Ther. 2019;10(1):339.

19. Shafiee A, Seyedjafari E, Soleimani M, Ahmadbeigi N, Dinarvand P, Ghaemi N. A comparison between osteogenic differentiation of human unrestricted somatic stem cells and mesenchymal stem cells from bone marrow and adipose tissue. Biotechnol Lett. 2011;33(6):1257-64.

20. Vishnubalaji R, Al-Nbaheen M, Kadalmani B, Aldahmash A, Ramesh T. Comparative investigation of the differentiation capability of bone-marrowand adipose-derived mesenchymal stem cells by qualitative and quantitative analysis. Cell Tissue Res. 2012;347(2):419-27.

21. Park SH, Sim WY, Min BH, Yang SS, Khademhosseini A, Kaplan DL. Chipbased comparison of the osteogenesis of human bone marrow- and adipose tissue-derived mesenchymal stem cells under mechanical stimulation. PLoS One. 2012;7(9):e46689.

22. Hsia CC. Comparative analysis of the mechanical signals in lung development and compensatory growth. Cell Tissue Res. 2017;367(3):687-705.

23. El Jamal A, Briolay A, Mebarek S, et al. Cytokine-induced and stretchinduced sphingosine 1-phosphate production by enthesis cells could favor abnormal ossification in spondyloarthritis. J Bone Miner Res. 2019;34(12): 2264-76.

24. Sarker MD, Naghieh S, McInnes AD, Schreyer DJ, Chen X. Regeneration of peripheral nerves by nerve guidance conduits: influence of design, biopolymers, cells, growth factors, and physical stimuli. Prog Neurobiol. 2018:171(3):125-50.

25. Koike M, Shimokawa H, Kanno Z, Ohya K, Soma K. Effects of mechanical strain on proliferation and differentiation of bone marrow stromal cell line ST2. J Bone Miner Metab. 2005;23(3):219-25.

26. Jagodzinski $M$, Breitbart $A$, Wehmeier $M$, et al. Influence of perfusion and cyclic compression on proliferation and differentiation of bone marrow stromal cells in 3-dimensional culture. J Biomech. 2008:41(9):1885-91.

27. Li YJ, Batra NN, You L, et al. Oscillatory fluid flow affects human marrow stromal cell proliferation and differentiation. J Orthop Res. 2004:22(6):1283-9.

28. Kramer N, Schmöllerl J, Unger C, et al. Autocrine WNT2 signaling in fibroblasts promotes colorectal cancer progression. Oncogene. 2017;36(39): 5460-72.

29. Jackson A, Vayssière B, Garcia T, et al. Gene array analysis of Wnt-regulated genes in C3H10T1/2 cells. Bone. 2005;36(4):585-98.

30. Wang Q, Liu H, Wang Q, et al. Involvement of c-Fos in cell proliferation, migration, and invasion in osteosarcoma cells accompanied by altered expression of Wnt2 and Fzd9. PLoS One. 2017;12:e0180558.

31. Park SJ, Bae HS, Park JC. Osteogenic differentiation and gene expression profile of human dental follicle cells induced by human dental pulp cells. J Mol Histol. 2015:46(1):93-106. 
32. Alexander MS, Kawahara G, Motohashi N, et al. MicroRNA-199a is induced in dystrophic muscle and affects WNT signaling, cell proliferation, and myogenic differentiation. Cell Death Differ. 2013;20(9):1194-208.

33. Hashemi Gheinani A, Burkhard FC, Rehrauer H, Aquino Fournier C, Monastyrskaya K. MicroRNA MiR-199a-5p regulates smooth muscle cell proliferation and morphology by targeting WNT2 signaling pathway. J Biol Chem. 2015;290(11):7067-86.

34. Qi B, Wang Y, Chen ZJ, et al. Down-regulation of miR-30a-3p/5p promotes esophageal squamous cell carcinoma cell proliferation by activating the Wnt signaling pathway. World J Gastroenterol. 2017;23(45):7965-77.

35. Chen PH, Liu AJ, Ho KH, et al. microRNA-199a/b-5p enhance imatinib efficacy via repressing WNT2 signaling-mediated protective autophagy in imatinib-resistant chronic myeloid leukemia cells. Chem Biol Interact. 2018; 291:144-51.

36. Wang Y, Cho C, Williams J, et al. Interplay of the Norrin and Wnt7a/Wnt7b signaling systems in blood-brain barrier and blood-retina barrier development and maintenance. Proc Natl Acad Sci U S A. 2018;115(50): E11827-36.

37. Chen $\mathrm{H}$, Ji X, Lee WC, et al. Increased glycolysis mediates Wnt7b-induced bone formation. FASEB J. 2019;33(7):7810-21.

38. Holguin N, Brodt MD, Silva MJ. Activation of Wnt signaling by mechanical loading is impaired in the bone of old mice. J Bone Miner Res. 2016;31(12): 2215-26.

39. Chen J, Tu X, Esen E, et al. WNT7B promotes bone formation in part through mTORC1. PLoS Genet. 2014;10(1):e1004145.

40. Zhang $\mathrm{H}$, Chen J, Shen $\mathrm{Z}$, et al. Indoxyl sulfate accelerates vascular smooth muscle cell calcification via microRNA-29b dependent regulation of Wnt/ $\beta$ catenin signaling. Toxicol Lett. 2018;284:29-36.

41. Yang Z, Sun Q, Guo J, et al. GRSF1-mediated MIR-G-1 promotes malignant behavior and nuclear autophagy by directly upregulating TMED5 and LMNB1 in cervical cancer cells. Autophagy. 2019;15(4):668-85.

42. Sun Y, Li L, Xing S, et al. miR-503-3p induces apoptosis of lung cancer cells by regulating p21 and CDK4 expression. Cancer Biomark. 2017;20(4):597-608.

43. Kim KS, Park Jl, Oh N, et al. ELK3 expressed in lymphatic endothelial cells promotes breast cancer progression and metastasis through exosomal miRNAs. Sci Rep. 2019;9(1):8418.

44. Zhao Z, Fan $X$, Jiang $L$, et al. miR-503-3p promotes epithelial-mesenchymal transition in breast cancer by directly targeting SMAD2 and E-cadherin. Genet Genomics. 2017:44(4):75-84.

45. Seo M, Kim SM, Woo EY, et al. Stemness-attenuating miR-503-3p as a paracrine factor to regulate growth of cancer stem cells. Stem Cells Int. 2018;2018:4851949.

46. Ma $Y$, Pan $X, X u P$, et al. Plasma microRNA alterations between EGFRactivating mutational NSCLC patients with and without primary resistance to TKl. Oncotarget. 2017:8(51):88529-36.

47. Assmann TS, Recamonde-Mendoza M, Costa AR, et al. Circulating miRNAs in diabetic kidney disease: case-control study and in silico analyses. Acta Diabetol. 2019;56(1):55-65.

48. Park J, Jeong S, Park K, Yang K, Shin S. Expression profile of microRNAs following bone marrow-derived mesenchymal stem cell treatment in lipopolysaccharide-induced acute lung injury. Exp Ther Med. 2018;15(6): 5495-502.

\section{Publisher's Note}

Springer Nature remains neutral with regard to jurisdictional claims in published maps and institutional affiliations.

Ready to submit your research? Choose BMC and benefit from:

- fast, convenient online submission

- thorough peer review by experienced researchers in your field

- rapid publication on acceptance

- support for research data, including large and complex data types

- gold Open Access which fosters wider collaboration and increased citations

- maximum visibility for your research: over $100 \mathrm{M}$ website views per year

At $\mathrm{BMC}$, research is always in progress.

Learn more biomedcentral.com/submissions 